\title{
Exceptionally potent human monoclonal antibodies are effective for prophylaxis and treatment of tetanus in mice
}

\author{
Marco Pirazzini, ${ }^{1}$ Alessandro Grinzato, ${ }^{1}$ Davide Corti, ${ }^{2}$ Sonia Barbieri, ${ }^{2}$ Oneda Leka, ${ }^{1}$ Francesca Vallese, ${ }^{1}$ Marika Tonellato, ${ }^{1}$ \\ Chiara Silacci-Fregni, ${ }^{3}$ Luca Piccoli, ${ }^{3}$ Eaazhisai Kandiah, ${ }^{4}$ Giampietro Schiavo, ${ }^{5,6}$ Giuseppe Zanotti, ${ }^{1}$ Antonio Lanzavecchia, ${ }^{3,7}$ \\ and Cesare Montecucco ${ }^{1,8}$ \\ DDepartment of Biomedical Sciences, University of Padova, Padova, Italy. ${ }^{2}$ Humabs BioMed SA, Bellinzona, Switzerland. ${ }^{3}$ Institute for Research in Biomedicine, Università della Svizzera Italiana, \\ Bellinzona, Switzerland. “European Synchrotron Radiation Facility, Grenoble, France. ${ }^{5}$ Department of Neuromuscular Diseases, Queen Square Institute of Neurology and ${ }^{6} U K$ Dementia Research Institute, \\ University College London, London, United Kingdom. ${ }^{7}$ Fondazione Istituto Nazionale Genetica Molecolare, Fondazione IRCCS Cà Granda Ospedale Maggiore Policlinico di Milano, Milano, Italy. ${ }^{8}$ Institute of \\ Neuroscience, National Research Council, Padova, Italy.
}

\begin{abstract}
We used human monoclonal antibodies (humAbs) to study the mechanism of neuron intoxication by tetanus neurotoxin and to evaluate these antibodies as a safe preventive and therapeutic substitute for hyperimmune sera to treat tetanus in mice. By screening memory B cells from immune donors, we selected 2 tetanus neurotoxin-specific mAbs with exceptionally high neutralizing activities and extensively characterized them both structurally and functionally. We found that these antibodies interfered with the binding and translocation of the neurotoxin into neurons by interacting with 2 epitopes, whose identification pinpoints crucial events in the cellular pathogenesis of tetanus. Our observations explain the neutralization ability of these antibodies, which we found to be exceptionally potent in preventing experimental tetanus when injected into mice long before the toxin. Moreover, their Fab derivatives neutralized tetanus neurotoxin in postexposure experiments, suggesting their potential for therapeutic use via intrathecal injection. As such, we believe these humAbs, as well as their Fab derivatives, meet the requirements to be considered for prophylactic and therapeutic use in human tetanus and are ready for clinical trials.
\end{abstract}

\section{Introduction}

Tetanus neurotoxin (TeNT) is a highly potent exotoxin responsible for tetanus, a life-threatening disease whose major symptoms are muscle rigidity and spasms, spastic paralysis, respiratory deficits, and autonomic dysfunction (1-5). TeNT is produced by toxigenic strains of the anaerobic sporogenic bacterium Clostridium tetani (6-8). The few amino acid variations found in the currently known TeNT isoforms do not change their immunogenic properties with respect to the prototypical TeNT (Harvard strain, E88; ref. 9).

TeNT consists of a light chain ( $\mathrm{L}, 50 \mathrm{kDa})$ and a heavy chain $(\mathrm{H}, 100 \mathrm{kDa})$ linked by a single interchain disulfide bond essential for neurotoxicity $(8,10)$. TeNT is generally described as a protein consisting of 3 domains, each of which serves a different step of its mechanism of neuronal intoxication. The carboxyterminal domain $\mathrm{HC}(50 \mathrm{kDa})$ is responsible for presynaptic binding and consists of 2 subdomains: the carboxy-terminal half

Authorship note: MP and AG contributed equally to this work

Conflict of interest: $A L, L P, C S$, and DC are employees of Vir Biotechnology, and $A L$ and DC hold shares in the company.

Copyright: @ 2021, American Society for Clinical Investigation.

Submitted: May 24, 2021; Accepted: September 28, 2021; Published: November 15, 2021. Reference information: / Clin Invest. 2021;131(22):e151676.

https://doi.org/10.1172/JCI151676.
(HC-C) contains a polysialoganglioside-binding (PSG-binding) site and a nidogen-binding site (11-14), whereas the aminoterminal half $(\mathrm{HC}-\mathrm{N})$ is essential for toxicity, although its function is not known (15). HC-N is linked to $\mathrm{HN}$ (50 kDa), the domain responsible for the membrane translocation of the $\mathrm{L}$ domain into the cytosol. The $\mathrm{L}$ domain is a metalloprotease that blocks neurotransmitter release (16).

C. tetani spores are ubiquitous in the environment and can contaminate necrotic wounds of any kind (burn, ulcer, surgical, tattoo, circumcision, needle injection, etc.), where spores may generate vegetative bacteria producing TeNT, which diffuses via the blood and lymphatic circulations. TeNT binds to motor, sensory, and autonomic presynaptic nerve terminals via at least 2 independent receptors: a PSG receptor and a protein receptor (5, 17). PSGs, including GT1b, GD1b, and GQ1b, play a major role in the initial membrane binding of TeNT (11-13), whereas nidogens 1 and 2 (also known as entactins 1 and 2) were identified as TeNT protein receptors (14). Nidogens and PSGs direct TeNT to presynaptic zones that, upon endocytosis, generate signaling endosomes containing neurotrophic factors and their receptors (18). These organelles undergo fast axonal retrograde transport to the perikaryon located in the spinal cord, where they release their contents (18-20). TeNT then binds to inhibitory interneurons and is endocytosed into the lumen of synaptic vesicles (SVs) (21). Acidification of the SV lumen induces a change in conformation of TeNT, 
whereby the HN domain inserts into the membrane and assists the membrane translocation of the L chain from the SV lumen to the cytosol. Here, the interchain disulfide bond is reduced by the thioredoxin reductase-thioredoxin redox system (22), releasing the metalloprotease activity of the L domain, which specifically cleaves the vesicle-associated membrane protein (VAMP) at a single site (23). Together with SNAP-25 and syntaxin, the VAMP forms a complex driving the fusion of SVs with the presynaptic membrane with ensuing neurotransmitter release (24).

VAMP cleavage in spinal cord inhibitory interneurons prevents the release of inhibitory neurotransmitters, which determines the hyperactivation of the postsynaptic motor neurons with sustained contraction of the innervated skeletal muscles, resulting in spastic paralysis $(25,26)$. Muscle contractures begin with trismus (lockjaw, a cardinal symptom of tetanus) and the neck, causing swallowing difficulty, and then descend to the thorax, causing respiratory deficit, generalized opposing skeletal muscle spasms, and autonomic dysfunctions that may lead to death (2).

Tetanus is prevented by a very effective vaccine based on tetanus toxoid that raises a long-lasting protection due to specific anti-TeNT antibodies, but after the age of 40 to 50 years, the anti-TeNT antibody titer decreases below TeNT neutralization levels (27). Tetanus is still a major killer in countries where appropriate public health care measures are not enforced, and where the infection of the umbilical cord stump or of the birth canal with nonsterile instruments causes neonatal and/or maternal tetanus (28).

Another common medical practice to prevent tetanus in patients presenting with necrotic wounds in hospital emergency rooms is the injection of anti-TeNT IgG, known as tetanus immunoglobulin (TIG), isolated from hyperimmune human donors (29). Hyperimmune horse sera are used in low-income countries because of their lower cost but can generate dangerous hypersensitivity reactions. TIG is also administered to patients with symptomatic tetanus to neutralize circulating TeNT and limit the severity of the disease, but this can cause problems (see Discussion; ref. 29). Intrathecal TIG administration is more effective, although this approach is limited by the low percentage of anti-TeNT antibodies present in TIG and by the amount of protein that can be injected into the cerebrospinal fluid $(30,31)$. All of these drawbacks can be overcome by using highly purified human monoclonal antibodies (humAbs), which are already approved for a variety of human diseases (32-38).

Here, we identified 2 humAbs with extremely high TeNT-neutralizing activity and determined the structure of their binding sites. These humAbs provided long-lasting prophylactic activity against lethal doses of TeNT, whereas their Fab fragments prevented tetanus in post-exposure experiments as effectively as TIG. These findings suggest that these humAbs and their Fab fragments represent appropriate and safe alternatives to TIG.

\section{Results}

HumAbs recognize distinct domains of TeNT and display distinct neutralizing capabilities. We isolated humAbs against TeNT from memory B cells obtained from immune adult donors who underwent booster vaccinations with tetanus toxoid as described previously (33). IgG memory B cells were immortalized under clonal condi- tions, and the antibodies produced in the culture supernatant were screened by ELISA for their ability to bind TeNT. We sequenced the antibody genes from 14 positive clonal cultures and produced IgG1 recombinant antibodies by transfecting HEK-293 cells.

To determine the TeNT domains recognized by the 14 humAbs, we performed Western blotting of the intact or reduced toxin and of the recombinant subdomains (Figure 1). The binding specificity of each antibody is shown in Supplemental Figure 1; supplemental material available online with this article; https://doi. org/10.1172/JCI151676DS1. Eleven antibodies bound to the HC domain, 3 to the $\mathrm{HN}$ domain, and none to the $\mathrm{L}$ chain. Three of the $11 \mathrm{HC}$-specific antibodies recognized the $\mathrm{HC}-\mathrm{C}$ part, and 8 of them bound to the HC-N portion (Supplemental Figure 1), suggesting that HC-N is the most immunogenic part of TeNT. Recent studies of anti-TeNT humAbs reported that the highest TeNT neutralization was displayed by antibodies recognizing HC, particularly those preventing PSG binding, but did not report on $\mathrm{HC}-\mathrm{N}$ recognition (39-42). Although the role of HC-N in neuron intoxication is currently unknown, its deletion causes loss of toxicity (15). Therefore, these specific humAbs could be very useful in unraveling the role of HC-N in the molecular and cellular pathogenesis of tetanus. Together with the knowledge that $\mathrm{HC}-\mathrm{C}$ contains the binding sites for the neuronal receptors of TeNT, these findings strongly suggest that the structure of TeNT is best described in terms of 4 domains ( $\mathrm{L}, \mathrm{HN}, \mathrm{HC}-\mathrm{N}$, and $\mathrm{HC}-\mathrm{C}$ ), rather than the 3-domain paradigm (L, HN, and $\mathrm{HC})$ reported so far $(8,43)$.

Inhibition of TeNT activity by humAbs. We tested humAbs in cerebellar granule neurons (CGNs) for their ability to inhibit TeNT cleavage of VAMP-2, an isoform highly expressed in CNS neurons. We chose CGNs because they are highly susceptible to TeNT, and VAMP cleavage is easily testable by immunoblotting and imaging (44). Figure 2A shows that an overnight incubation of CGNs with $50 \mathrm{pM}$ TeNT was sufficient to cleave all the VAMP-2 of these neurons. An initial screening with a hundred-fold molar excess showed that only 4 of the 14 humAbs tested (TT104, TT110, TT109, and TT39) were able to prevent TeNT action, albeit to different extents. Accordingly, we focused on these humAbs and tested various humAb/TeNT molar ratios to determine their neutralizing potency. As shown in Figure 2B, TT39 and TT109 showed partial neutralization. In contrast, both TT104 and TT110 prevented TeNT intoxication already at a low molar ratio, with TT104 being the most powerful. These results were paralleled by immunofluorescence staining for VAMP-2 (Figure 2C).

We then tested the selected humAbs for their ability to neutralize TeNT in vivo. Figure 2D shows that preincubation of TeNT with TT104 and TT110 prevented the development of tetanus in mice in a dose-dependent manner, with TT104 displaying full neutralization at an equimolar ratio. On a molar basis, we found that TT110 was less effective, but reduced tetanus symptoms at a 2.5:1 molar ratio and completely neutralized TeNT at a 5:1 ratio. TT39 and TT109 did not protect mice from TeNT challenge. Accordingly, we chose TT104 and TT110 for further structural and functional analyses.

Structure of the ternary complex of TeNT with TT104 and TT110 Fab fragments. Recombinant Fab fragments of TT104 and TT110 were produced by introducing a stop codon after the $\mathrm{C}_{\mathrm{H}} 1$ domain (Supplemental Figure 2). The dissociation constants of 
A

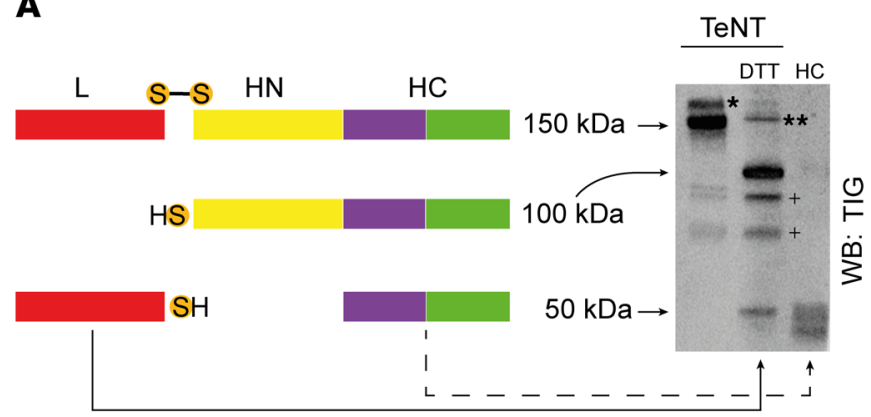

B

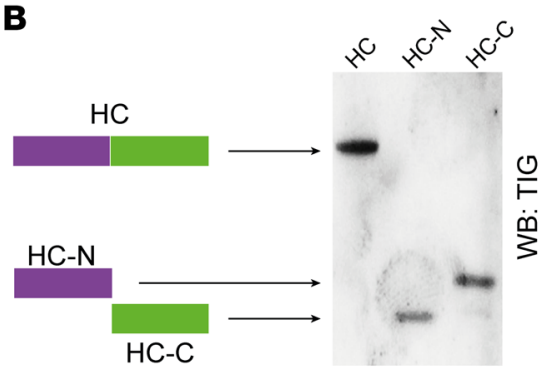

\begin{tabular}{|c|c|c|c|c|c|}
\hline \multirow{2}{*}{ Antibody } & \multirow{2}{*}{ TeNT } & \multicolumn{2}{|c|}{ Reduced TeNT } & \multirow{2}{*}{$\begin{array}{c}\text { Recombinant } \\
\text { HC }\end{array}$} & \multirow{2}{*}{$\begin{array}{c}\text { Domain } \\
\text { specificity }\end{array}$} \\
\hline & & L chain & $H$ chain & & \\
\hline $\begin{array}{c}\text { Human } \\
\text { antiserum } \\
\end{array}$ & + & + & + & + & Whole toxin \\
\hline TT 4 & + & & + & + & $\mathrm{HC}$ \\
\hline ТT 13 & + & & + & + & $\mathrm{HC}$ \\
\hline TT 16 & + & & + & + & $\mathrm{HC}$ \\
\hline Tा 33 & + & & + & & $\mathrm{HN}$ \\
\hline ТT 39 & + & & + & + & $\mathrm{HC}$ \\
\hline TT 102 & + & & + & + & $\mathrm{HC}$ \\
\hline TT 103 & + & & + & + & $\mathrm{HC}$ \\
\hline TT 104 & + & & + & + & $\mathrm{HC}$ \\
\hline TT 107 & + & & + & + & $\mathrm{HC}$ \\
\hline TT 108 & + & & + & + & $\mathrm{HC}$ \\
\hline TT 109 & + & & + & + & $\mathrm{HC}$ \\
\hline TT 110 & + & & + & & $\mathrm{HN}$ \\
\hline TT 114 & + & & + & + & $\mathrm{HC}$ \\
\hline TT 115 & + & & + & & $\mathrm{HN}$ \\
\hline
\end{tabular}

D

\begin{tabular}{c|c|c|c|c} 
Antibody & $\begin{array}{c}\text { Recombinant } \\
\text { HC }\end{array}$ & $\begin{array}{c}\text { Recombinant } \\
\text { HC-N }\end{array}$ & $\begin{array}{c}\text { Recombinant } \\
\text { HC-C }\end{array}$ & $\begin{array}{c}\text { Subdomain } \\
\text { specificity }\end{array}$ \\
\hline $\begin{array}{c}\text { Human } \\
\text { antiserum }\end{array}$ & + & + & + & Whole HC \\
\hline TT 4 & + & + & + & HC-N \\
\hline TT 13 & + & HC-C & HC-N \\
\hline TT 16 & + & + & & HC-N \\
\hline TT 39 & + & + & & HC-C \\
\hline TT 102 & + & + & + & HC-N \\
\hline TT 103 & + & + & & HC-C \\
\hline TT 104 & + & & + & HC-N \\
\hline TT 107 & + & + & & HC-N \\
\hline TT 108 & + & + & & HC-N \\
\hline TT 109 & + & + & & HC-N \\
\hline TT 114 & + & + & &
\end{tabular}

Figure 1. TeNT polypeptide chains and domain-specific recognition by TT humAbs. (A) Schematic structure of TeNT (top panel) and TeNT-HC (middle panel). Bottom panel shows the L chain (red) and HC subdomains HC-N (violet) and HC-C (green) and as they appear in Western blotting (WB) using TIC as the primary antibody. Whole TeNT has a molecular weight of $150 \mathrm{kDa}$, corresponding to the $\mathrm{L}$ chain plus the $\mathrm{H}$ chain (left line). Reduction with DTT generated 2 bands corresponding to $\mathrm{HC}$ ( $\mathrm{HN}$ plus $\mathrm{HC}, 100 \mathrm{kDa}$ ) and L ( $50 \mathrm{kDa}$ ). Recombinant HC has a molecular weight of approximately $50 \mathrm{kDa}$. The L chain has a weak signal, possibly due to a low immunoreactivity of L-specific IgGs in TIG. Single asterisk indicates a redox isomer of TeNT; double-asterisk indicates single-chain TeNT; plus sign indicates degradation fragments. (B) Schematic structure of HC (top), HC-N (middle), and HC-C (bottom) and how they appear in Western blotting stained for TIG. (C and D) Summary tables of recognition of TeNT domains and subdomains by TT humAbs as detected by Western blotting. The specificity of TT-humAbs was determined by at least 3 independent trials per antibody.

the toxin-Fab complexes were measured by surface plasmon resonance using immobilized TeNT as a bait and found to be $6.7 \times 10^{-12} \mathrm{M}$ and $3.0 \times 10^{-9} \mathrm{M}$ for TT104-Fab and TT110-Fab, respectively (Supplemental Figure 3). The high affinity of these interactions suggested the possibility that stable binary and ternary complexes could be formed between TeNT and the 2 Fabs. Indeed, immunocomplexes were obtained by incubation of single components in either binary (1:1) or ternary mixtures (1:1:1) (Supplemental Figure 4). The ternary [TeNT]-[TT104-Fab][TT110-Fab] immunocomplex was purified to homogeneity by gel filtration (total mass $250 \mathrm{kDa}$ ). Attempts to crystallize this complex were not successful, and therefore we switched to cryo- electron microscopy (cryo-EM) that produced a model fitting the available structure of TeNT (Protein Data Bank [PDB] ID: 5nOb) and of the 2 Fabs modeled using the sequences of the TT10 4 and TT110 Fabs. The overall 4-domain folding of TeNT was well conserved in the immunocomplex and superimposed with the structures of isolated domains (Figure 3, A and B) and of the whole toxin (Figure 3, C and D, and refs. 8, 45-47).

Similar to TeNT alone (8), we found that the trimeric complex [TeNT]-[TT104-Fab]-[TT110-Fab] in solution was flexible (Supplemental Figure 5A). The flexibility was intrinsic to TeNT and was not influenced by the binding of the Fabs; it was mainly located around residues 870-875, a loop not resolved in the crys- 
A

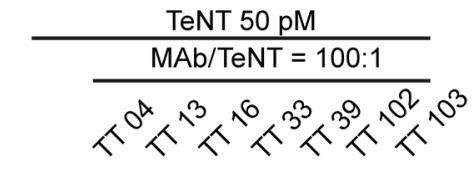

SNAP-25

VAMP-2

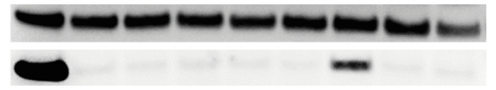

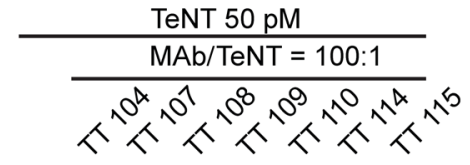

SNAP-25

VAMP-2
B

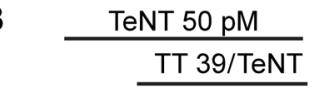

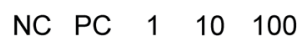

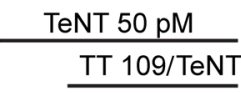

NC PC $150 \quad 100$

SNAP-25

VAMP-2

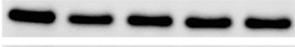

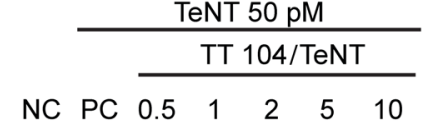

$\begin{array}{lllllll}\mathrm{NC} & \mathrm{PC} & 0.5 & 1 & 2 & 5 & 10\end{array}$

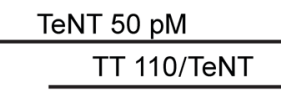

$\begin{array}{llllll}\text { NC } & P C & 1 & 5 & 10 & 50\end{array}$
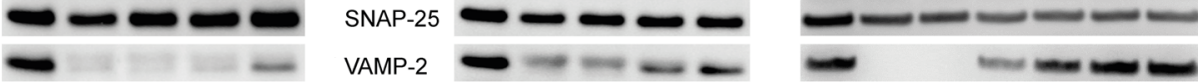

SNAP-25

VAMP-2

C

TeNT 50 pM
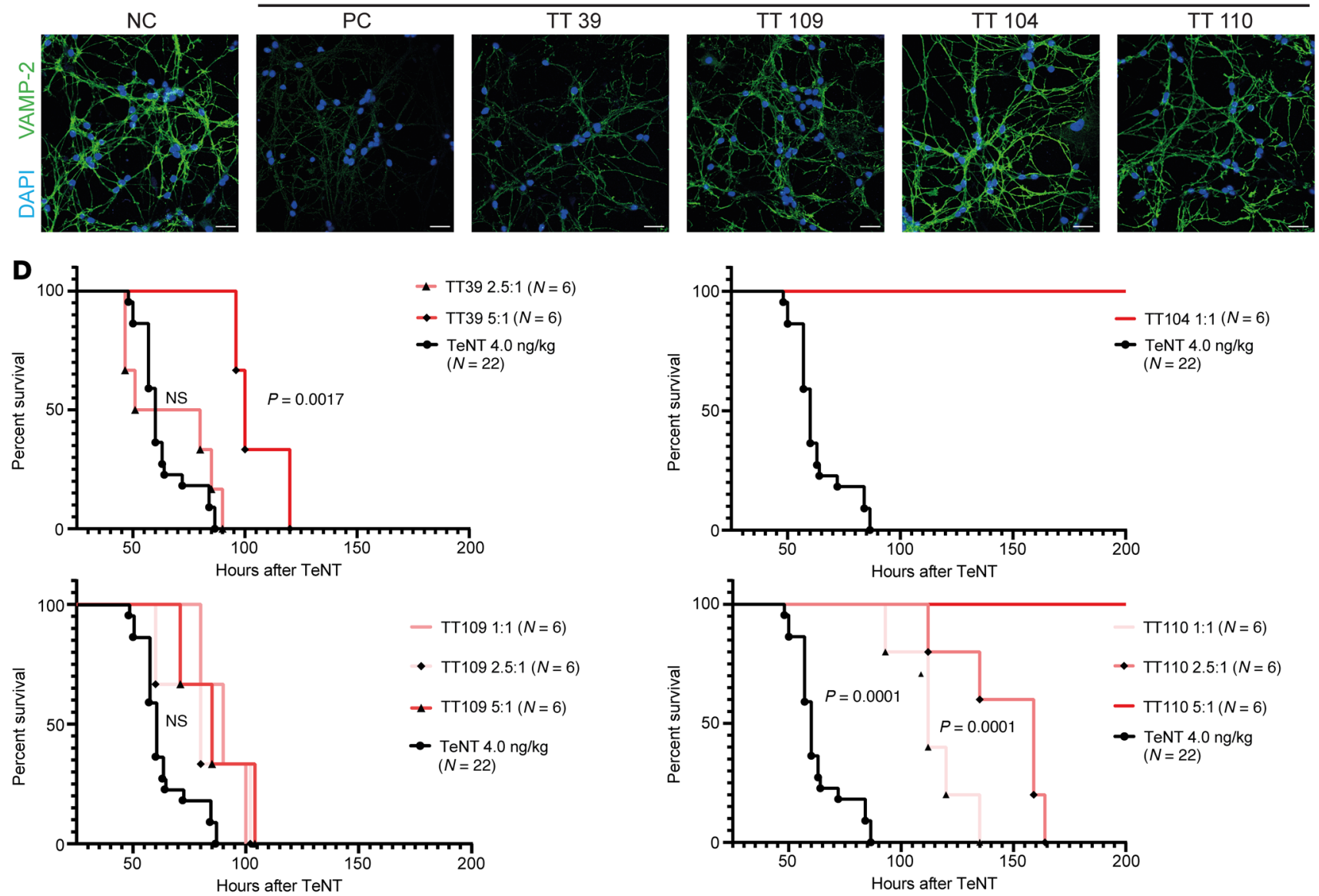

Figure 2. Preliminary screening for TeNT neutralization by TT humAbs assayed in vitro and in vivo. (A) TeNT ( 50 pM) was diluted in complete culture medium alone (positive control) or supplemented with a 100X molar excess of the indicated humAb. The mixture was then added to CGNs for 12 hours, and TeNT activity was evaluated by monitoring the cleavage of VAMP-2 with an antibody recognizing only the intact form. SNAP-25 was used as a loading control. (B) Effect of different TeNT/humAbs ratios for the humAbs displaying toxin-neutralizing activity on CGNs. (C) Immunofluorescence analysis performed with an antibody specific for intact VAMP-2 (green) to assay for the TeNT-neutralizing activity of TT39, TT104, TT109, and TT110 preincubated with TeNT (100:1 molar ratio) and added to the primary culture of CGNs. Control CGNs (NC) are labeled in green, whereas neurons treated with TeNT alone (PC) do not display this signal because of the complete cleavage of VAMP-2. CGNs treated with the indicated humAbs displayed intermediate signals depending on the neutralization activity of the humAbs. Images are representative of 3 independent experiments. Scale bars: $25 \mu \mathrm{m}$. (D) Mice were injected i.p. with TeNT (4 ng/kg, black trace) alone or preincubated with the indicated molar ratios of humAb/TeNT, and survival is plotted as a function of time after toxin injection. $P$ values are shown in the panels and were determined by Mantel-Cox test. The number of mice in each group is indicated in the panels. NC, negative control; PC, positive control. 
tal structure of the toxin and corresponding to the connection between the C-terminal of HN and the N-terminal of HC-N (8).

To overcome the flexibility of the complex, the structure was split into 2 parts analyzed separately, and 2 masks were generated by the multibody technique to yield 2 different maps, one relative to [TeNT-L-HN]-[TT110-Fab], and the other one to [TeNT-HC][TT104-Fab].

Structure of [TeNT-L-HN]-[TT11O-Fab]. Owing to the preferred orientation assumed by the particles in the grid, our analysis of the [TeNT-L-HN]-[TT110-Fab] portion provided a resolution of 8.3 $\AA$, which was insufficient to define their interactions at the atomic level. However, the overall shapes could be clearly distinguished and showed that TT110-Fab binds the HN domain opposite the $\mathrm{L}$ domain (Figure $3 \mathrm{~A})$. The buried surface of this epitope is estimated to be $700 \AA^{2}$ and $380 \AA^{2}$ for chains $V_{H}$ and $V_{L}$, respectively, and the interaction area involves the TeNT helices 597-607 and 614-625, extending to the following strand until residue 631 and including segment 655-663. Importantly, this latter segment is part of the "BoNT-switch," a structural module proposed to be a main driver of the low-pH-induced membrane insertion of $\mathrm{HN}$ in the botulinum neurotoxins (BoNTs), a group of toxins sharing the structural architecture and the mechanism of neuron intoxication of TeNT (48). The BoNT switch is composed of disordered loops and 3 short helices $\left(\alpha_{A}, \alpha_{B}, \alpha_{C}\right)$ that are also present in TeNT (Supplemental Figure 7); TT110-Fab binds to a region in TeNT corresponding to the $\alpha_{B}$ of BoNT serotype A1 (BoNT/A1) (Supplemental Figure 8). At acidic $\mathrm{pH}$, the BoNT/A1 switch rearranges into $5 \beta$ strands (dubbed $\beta 1-\beta 5$ ), with $\alpha_{A}$ flipping out from the toxin structure at the center of an elongated hinge formed by $\beta 2 / \beta 3$ hairpins (corresponding to residues I630-Y648 in BoNT/A1 and to V639Y657 in TeNT). This hinge was shown to insert into the lipid bilayer and to be essential for the subsequent membrane translocation of the L domain of BoNT/A1 (48). At the same time, $\alpha_{B}$ and $\alpha_{C}$ form the $\beta 4 / \beta 5$ hairpin with a hydrophobic surface generated by the structural rearrangement. Considering that TT110-Fab binds to $\alpha_{B}$, it is very likely that it neutralizes TeNT by interfering with the low-pH-driven insertion of $\mathrm{HN}$ into the membrane, thus blocking the translocation of the $\mathrm{L}$ domain into the cytosol.

Structure of [TeNT-HC]-[TT1O4-Fab]. The map of the portion of the complex including TT104-Fab and the HC-N and HC-C domains (residues $875-1110$ and 1111-1315, respectively) has an overall resolution of $3.9 \AA$. The area of interaction of the antibody with the HC-C domain is opposite the $\mathrm{HN}$ and L domains and includes portions of polypeptide strands 1140-1145, 1149-1157, $1171-1173,1202-1204$, and $1276-1281$ (Figure 4). From the Fab side, the interaction involves mainly the $\mathrm{V}_{\mathrm{H}}$ chain, whose surface area buried upon the binding is $555 \AA^{2}$, while the buried area of chain $V_{L}$ is $396 \AA^{2}$. This difference is reflected in the number of interactions. $\mathrm{V}_{\mathrm{H}}$ residues form 1 salt bridge and 7 potential hydrogen bonds, whereas $\mathrm{V}_{\mathrm{L}}$ contributes to stabilize the protein-protein interaction with 4 additional hydrogen bonds (Supplemental Table 1, calculation performed with the PISA server; ref. 49). The total TeNT buried surface in the complex with TT104-Fab is $792 \AA^{2}$.

The TT104-Fab binding site is only $12 \AA$ away from the putative nidogen-binding site modeled previously (14), and its proximity suggests a possible interference with the binding of nidogen to $\mathrm{HC}-\mathrm{C}$ due to a steric clash (Supplemental Figure 6). At the same time, this binding might alter the ability of HC-C to interact with the oligosaccharide portion of PSG, which projects out of the neuronal plasma membrane and has to be accommodated into the HC-C to mediate binding (17). These possibilities were tested experimentally (see below).

Model of the overall structure of the immunocomplex. Owing to the intrinsic flexibility of the complex in solution, it was not possible to obtain a complete 3D map of the entire immunocomplex. Nevertheless, thanks to the available crystal structure of TeNT (8, 39), we built a model of the ternary complex by superimposing the L, HN, HC-N, and HC-C domains over the TeNT crystal structure (Figure 3C). The flexibility of the TeNT-Fabs complex observed in our images is intrinsic to TeNT (8), and, accordingly, this model represents one of the possible conformations of the toxin with the 2 Fabs bound. We detected several eigenvalues (Supplemental Figure 5, B and C) that defined the dynamics of the motion of the L-HN domains relative to the HC-N and HC-C domains. All the eigenvalues were unimodal, suggesting that these motions are continuous. The first 3 eigenvalues described $85 \%$ of the variability present in the data set. The movies of the reconstructed body repositioned along these eigenvectors revealed that the 2 domains of TeNT have a rocking motion (Supplemental Videos 1, 2, and 3). Interestingly, the conformation of TeNT in the immunocomplex was closer to the open conformation of the full-length toxin, in which the C869-C1093 disulphide bond was reduced (39), than to that of the nonreduced TeNT (8).

TT104-Fab inhibits the biological activity of TeNT by preventing membrane binding. TT104-Fab prevented cleavage of VAMP in CGNs and the development of tetanus in mice (Figure 5, A and B). The TeNT-TT104-Fab interaction suggests that this Fab should interfere with toxin binding. As detected using a fluorescently labeled HC fragment (A555-TeNT-HC, red signal in Figure 5, C and D), TT104-Fab prevented the binding of TeNT to CGNs and to the neuromuscular junction (NMJ). TT104-Fab inhibition was very specific, as indicated by the lack of interference with the binding of a fluorescent HC of BoNT/A1 (CpV-BoNT/A1-HC, green signal in Figure 5, C and D). Of note, the CpV-BoNT/A1-HC signal was slightly brighter in the presence of TT104-Fab, which sequestered A555-TeNT-HC, preventing the competition of the 2 toxin HCs for PSG binding (green signals in Figure 5, C and D).

To elucidate the specific mechanism responsible for the TT104-Fab-mediated inhibition of TeNT binding, we assayed the interaction of TeNT with immobilized GT1b, purified nidogen-1, nidogen-2, or their combinations. Figure $5 \mathrm{E}$ shows that TeNT binding to GT1b was completely prevented by TT104-Fab, while binding to the nidogens was strongly reduced. This result is in line with the definition of the surface of the conformational epitope recognized by the idiotype of TT104-Fab. Interestingly, when GT1b and nidogens were immobilized together (Figure 5E, right panel), TeNT binding in the presence of TT104-Fab compared with that reached when only nidogens were present. These findings support the model in which nidogen-TeNT interaction occurs at a distinct site with respect to that of PSG (14) and indicate that TT104-Fab interfered with the binding of both receptors. At the same time, these results clearly indicate that membrane binding was the specific step inhibited by TT104 and TT104-Fab, with a consequent strong anti-tetanus activity in vivo. 
A

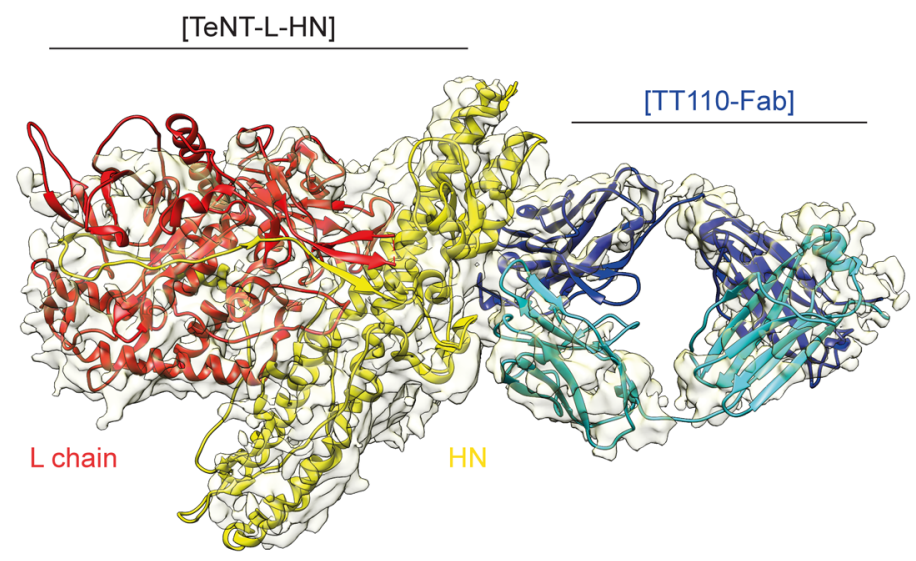

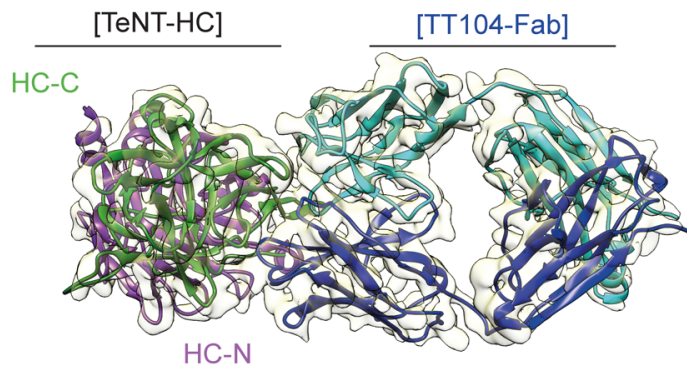

B

Structure of [TeNT-HC] - [TT104-Fab]
C

Structure of [TeNT] - [TT104-Fab] - [TT110-Fab]

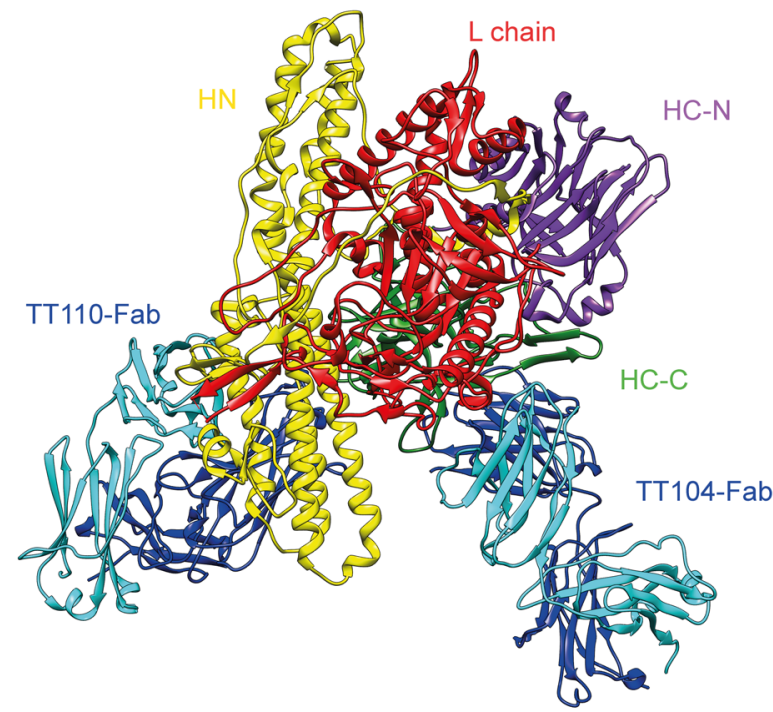

D
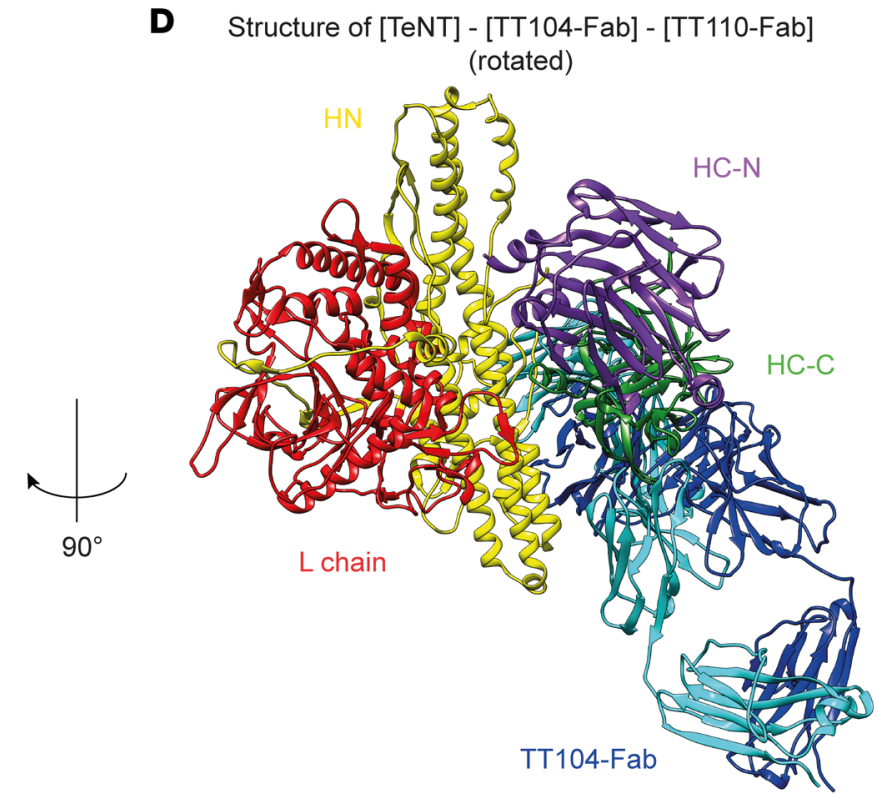

Figure 3. Cryo-EM structure of the [TeNT-HC]-[TT104-Fab]-[TT110-Fab] ternary complex. (A) Structure of HN (yellow) and L (red) domains in complex with TT110-Fab (light and dark blue for the variable L and H chains, respectively). (B) Structure of the HC-C (green) and HC-N (purple) domains in complex with TT104-Fab (light and dark blue for the variable L and H chains, respectively). (C and D) Overall structure of the TeNT-Fabs complex colored as in A and B.

TT110-Fab inhibits the low-pH-induced conformational change of TeNT. Although less powerful than TT104-Fab, the HN-specific TT110-Fab also effectively prevented the cleavage of VAMP by TeNT in cultured neurons (Figure 6A) and protected mice from TeNT challenge when the toxin and the Fab were preincubated together before i.p. injection (Figure 6B). As discussed above, the structure of the immunocomplex suggests that TT110 interfered with the $\mathrm{HN}$-dependent membrane translocation of the $\mathrm{L}$ chain driven by acidification. After neurotransmitter release, SVs are endocytosed and acidified by the vacuolar ATPase, a process necessary for neurotransmitter reloading. TeNT inside SVs in central neurons (21) exploits this physiological process to change structure with exposure of hydrophobic patches on $\mathrm{HN}$ that mediate its membrane insertion in such a way as to assist the membrane translocation of the L domain (50). As this process cannot be experimentally accessed from outside the cells, we took advan- tage of a method previously devised to induce low-pH membrane translocation of the $\mathrm{L}$ chain directly from the plasma membrane into the cytosol (50-53). As schematized in Figure 6C, after TeNT binding to neurons at $0^{\circ} \mathrm{C}$, membrane translocation is induced by replacing the cold medium with acidic medium at $37^{\circ} \mathrm{C}$ for a few minutes. Neurons are then incubated in control medium in the presence of bafilomycin A1 (Baf-A1), a v-ATPase inhibitor preventing toxin entry through the canonical route, and L domain translocation is assessed by determining VAMP-2 cleavage. Figure 6D shows that upon exposure to $\mathrm{pH}$, the L metalloprotease entered the cytosol and efficiently cleaved VAMP-2. In contrast, when TeNT was preincubated with TT110-Fab, VAMP-2 was no longer cleaved, consistent with a block of translocation.

To test the possibility that TT110-Fab prevents the low-pHinduced structural change of TeNT $(51,54,55)$, we performed a fluorometric assay based on the binding of the lipophilic dye 8- 
A

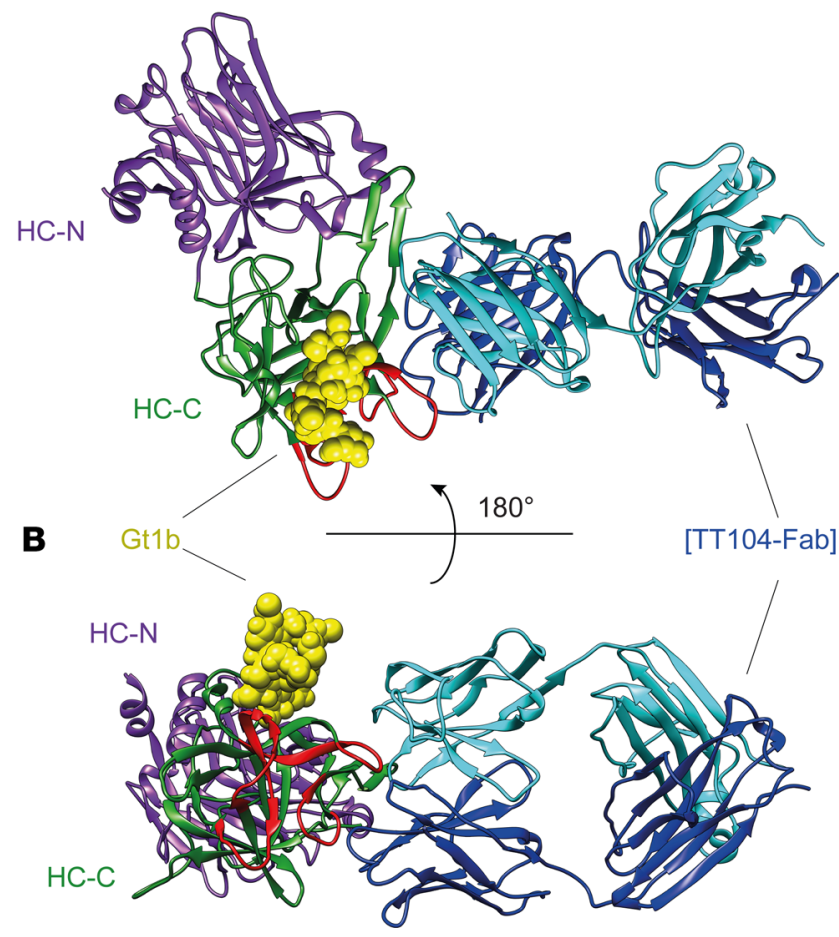

Figure 4. Model of [TeNT-HC]-[TT104-Fab] interaction in complex with GT1b. (A) The HC-N (purple) and HC-C (green) domains of TeNT complexed with TT104-Fab (light and dark blue), and the oligosaccharide portion of GT1b (yellow; bound as in pdb:1FV3). The nidogen binding region is shown in red. (B) View of the model after $180^{\circ}$ horizontal rotation.

anilino-1-naphthalenesulfonic acid (ANS) to hydrophobic protein patches (56). Figure 6E shows that TT110-Fab prevented TeNT from undergoing the low-pH-driven conformational change with exposure of hydrophobic surface patches. This result supports the hypothesis that TT110 blocks the "BoNT/TeNT-switch" of HN and prevents the occurrence of low-pH-driven insertion of $\mathrm{HN}$ and the subsequent membrane translocation of the $\mathrm{L}$ domain). Given these results and the available literature, we propose that the protonation of Asp618, Asp621, Asp622, Glu626, Glu658, and Glu666 of TeNT (Supplemental Figure 8) is likely to be the key initial event of the low-pH conformational transition of HN leading to its membrane insertion. Future mutagenesis experiments targeting these residues will provide conclusive evidence for the role of these acidic sites in the molecular pathogenesis of tetanus.

Collectively, the above experiments demonstrate that TT110Fab and TT104-Fab acted on 2 different steps of the process of TeNT nerve terminal intoxication, predicting that they should display additive effects in preventing experimental tetanus in vivo.

Prophylaxis and therapy in experimental tetanus by TT104, TT110, and their Fabs. The most frequently used protocol for testing the neutralizing activity of anti-TeNT antibodies involves their preincubation with TeNT. This procedure does not match the conditions of injured patients, for whom anti-TeNT IgG antibodies are used as tetanus prophylactic agents before the toxin is produced by C. tetani in necrotic wounds. Therefore, we performed a set of experiments in which mice were pretreated with a single i.p. injection of TT104 and TT110, alone or in combination, before inoculation with five $50 \%$ mouse lethal doses $\left(\mathrm{MLD}_{50}\right)$ of TeNT at delayed time points. Since tetanus usually develops with incubation times ranging from 2 to 15 days after injury $(1,2)$, the animals were pretreated with TT104 or TT110 alone (400 ng/kg each) or in combination ( $200 \mathrm{ng} / \mathrm{kg}$ plus $200 \mathrm{ng} / \mathrm{kg}$ ), 7 and 15 days before TeNT injection (Figure 7A). As a control, we pretreated a cohort of animals with TIG (7 IU/kg, corresponding to the standard prophylactic dose of 500 IU used in a human weighing 70 $\mathrm{kg}$ ). Remarkably, we found that TT104 provided the mice with full protection from tetanus, even when they were injected 15 days before TeNT inoculation (Figure 7B). TT110 was less effective, since it was fully protective only when a challenge was given on day 7 (Figure 7C). The combination of $200 \mathrm{ng} / \mathrm{kg}$ TT104 and of $200 \mathrm{ng} / \mathrm{kg}$ TT110 provided full protection of mice for 15 days, as was observed with TIG treatment (Figure 7D).

Once the metalloprotease domain of TeNT has been released inside the cytoplasm of target neurons, the toxin can no longer be neutralized by anti-TeNT antibodies. However, worsening of the symptoms can be prevented by neutralization of circulating TeNT, and this is the rationale supporting the general practice of injecting TIG into hospitalized patients who show symptoms of tetanus. Thus, TIG and TT104 or TT110 were compared in a therapeutic setting for their capacity to interfere with tetanus at different time points after TeNT challenge. We opted to use the Fab derivatives in consideration of their possible use by intrathecal rather than peripheral administration. To properly compare the Fabs and TIG, we estimated the concentration of anti-tetanus-specific IgG present in the standard dose of $500 \mathrm{IU}$ (in a putative patient weighing $70 \mathrm{~kg}$ ) on the basis of previous quantifications of serum from hyperimmunized human donors (57). As shown in Figure 7E, TeNT inoculation ( $4 \mathrm{ng} / \mathrm{kg}$ ) was followed by injection of either TIG or the corresponding amount of TT104-Fab and TT110-Fab in combination after different time periods (1.5, 3, 6, and 12 hours). Tables 1 and 2 show that the Fab combination, like TIG, completely protected mice up to 6 hours after injection of TeNT. However, neither form of serotherapy blocked the toxin completely when injected 12 hours after TeNT challenge, in agreement with what is known about the kinetics of TeNT internalization in neurons (5). Notably, in this case, mice developed tetanus symptoms over a similar time course (Figure 7F), further indicating that the Fab combination and TIG are comparably effective in neutralizing the activity of the toxin present in body fluids.

\section{Discussion}

Here, we report the structural and functional characterization of 2 humAbs that prevented tetanus by neutralizing TeNT at near-stoichiometric antibody/toxin ratios, a property maintained by their Fabs. TT104 interacted strongly with the HC-C domain that mediates TeNT binding to neurons, while TT110 bound the HN domain that mediates translocation of the metalloprotease domain into the neuronal cytosol.

Cryo-EM revealed the structure of the [TeNT]-[TT104-Fab][TT110-Fab] trimeric immunocomplex, and we were able to identify the 2 TeNT epitopes. This finding explains why these antibodies were so potent in preventing tetanus in mice, and also after 
A

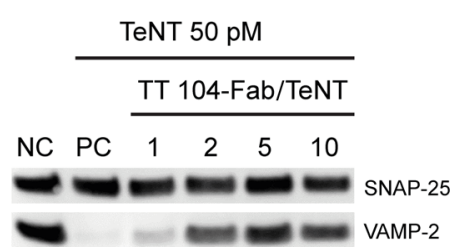

B

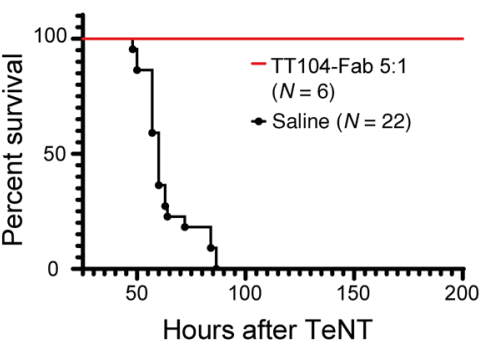

C
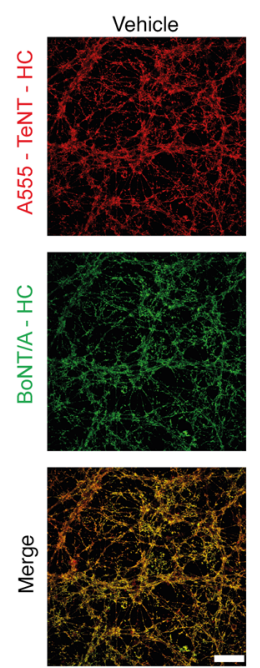

TT104-Fab
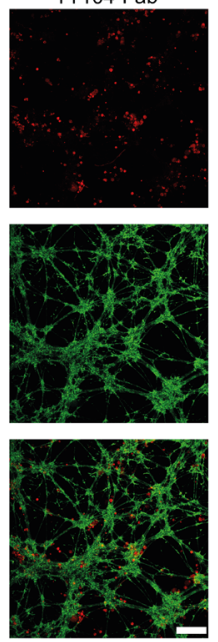

D
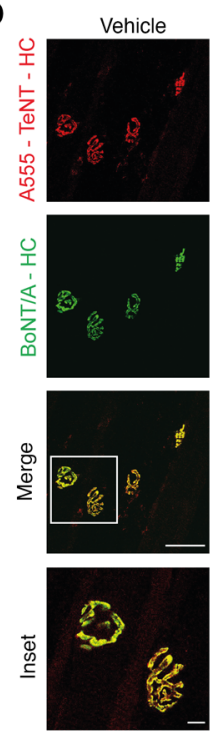

TT104-Fab
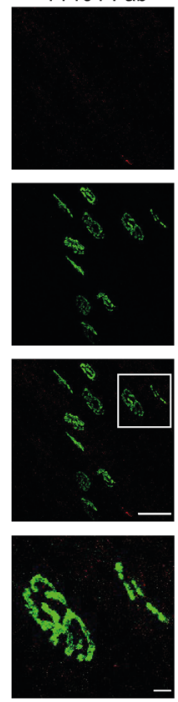

\section{E}
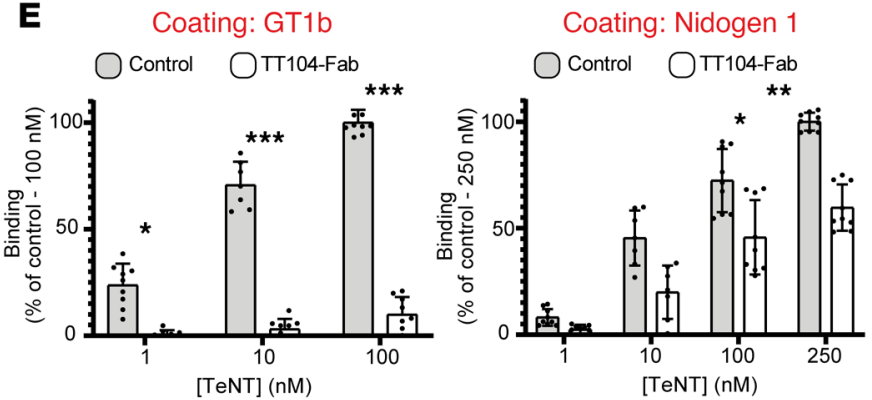

Coating: Nidogen 1
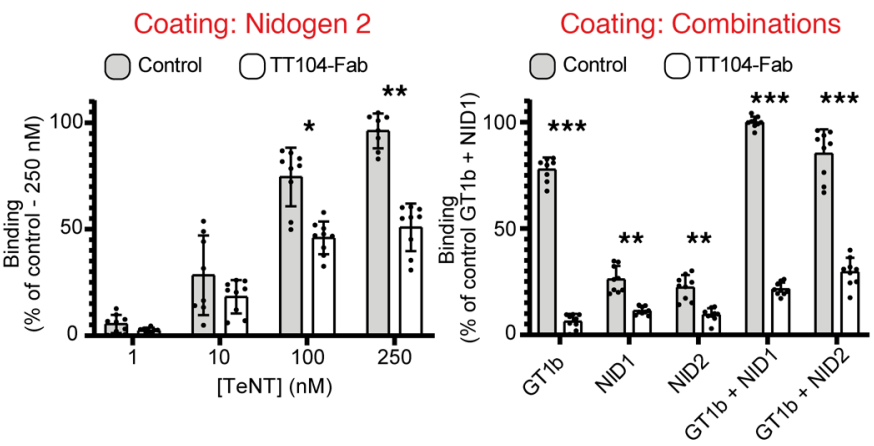

Figure 5. TT104-Fab prevents TeNT toxicity by interfering with toxin binding to PSGs and nidogen. (A) Western blot analysis of CGNs treated with $50 \mathrm{pM}$ TeNT alone (PC) or preincubated with the indicated TT104-Fab/TeNT molar ratios. After 12 hours, TeNT activity was evaluated by monitoring the cleavage of VAMP-2, as in Figure 2. Data are representative of 3 independent experiments. (B) Survival of mice injected i.p. with either TeNT alone (4 ng/kg) or premixed with a 5:1 molar ratio TT104-Fab/TeNT. The number of mice in each group is shown in the panel. (C) Fluorescence in CGNs treated with a mixture of $50 \mathrm{nM}$ A555-TeNT-HC (red) or $50 \mathrm{nM} \mathrm{CpV-BoNT/A-HC} \mathrm{(BoNT/A-HC,} \mathrm{green)} \mathrm{preincubated} \mathrm{with} \mathrm{either} \mathrm{culture} \mathrm{medium} \mathrm{or} \mathrm{a} \mathrm{2:1} \mathrm{molar} \mathrm{ratio} \mathrm{of} \mathrm{TT104-Fab/TeNT-HC} \mathrm{for}$ 2 hours and observed with a confocal microscope. Images are representative of 3 independent experiments. Scale bars: $30 \mu \mathrm{m}$. (D) Immunofluorescence staining of the LAL muscle injected in vivo with A555-TeNT-HC $(1 \mu \mathrm{g})$ or CpV-BoNT/A-HC $(1 \mu \mathrm{g})$ preincubated with vehicle or a 2:1 molar ratio of TT104-Fab/ TeNT and observed 2 hours later with a confocal microscope. Images are representative of 3 independent experiments. Scale bars: $50 \mu \mathrm{m} ; 10 \mu \mathrm{m}$ (enlarged insets). (E) Purified GT1b (0.5 g/well, left panel), recombinant nidogen-1/-2 (250 ng/well, middle left and middle right panels), or their combination (right panel) were adsorbed by overnight incubation on ELISA plates, and the binding of the indicated concentrations of either TeNT alone (gray bars) or TeNT preincubated with TT110-Fab (white bars) was tested as described previously (12). Data are reported as the percentage of the highest value in the graph and were averaged from at least 3 independent experiments (each dot represent a single well). Data represent the mean $\pm \mathrm{SD}$. ${ }^{*} P<0.05$, ${ }^{* *} P<0.01$, and ${ }^{* * *} P<$ 0.001 , by 2 -tailed $t$ test.

being administered up to 15 days before TeNT treatment. These results are even more relevant considering that the lifetime of human IgGs in mice is expected to be lower than that in humans $(58,59)$, leading us to predict that the duration of strong protection conferred by TT104 and TT110 will be even longer in humans. In addition, it should be considered that these antibodies could be engineered to achieve an extended half-life and biodistribution (60). Remarkably, TeNT neutralization was effective in the ng/ $\mathrm{kg}$ range, a dose orders of magnitude lower than the $\mathrm{mg} / \mathrm{kg}$ range of TIG, offering the possibility of increasing the dose if necessary.

To the best of our knowledge, this is the first report showing such potent and long-lasting prophylactic effectiveness of antiTeNT humAbs that fully meets the needs of patients entering hospital emergency rooms with necrotic wounds possibly contami- nated by C. tetani spores. TT104 and TT110 are also expected to be very effective in preventing maternal and/or neonatal tetanus in cases of delivery by a mother not immunized against tetanus, a frequent condition in several parts of the world (28).

The current serotherapy using IgG isolated from the blood of hyperimmune donors has several drawbacks: (a) variation of neutralizing strength from lot to lot; (b) possible risk of contamination with unknown viruses or blood proteins; (c) requirement of injecting relatively large amounts of proteins (typically $16 \%-20 \% \mathrm{w} / \mathrm{v}$ ), which may elicit adverse reactions such as angioedema or anaphylaxis; (d) high risk of anaphylactic reactions or serum sickness in the case of horse antisera; (e) patients with an IgA deficit could mount an immune response to the small amount of IgA present in TIG. Pain at the site of injection as well as fever, dizziness, and oth- 
A

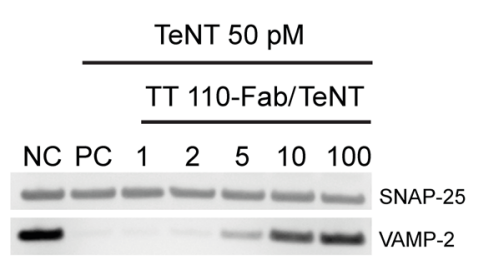

B

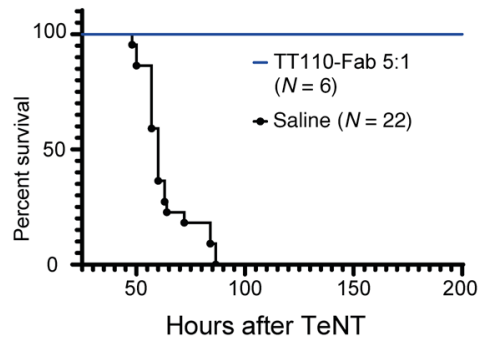

C

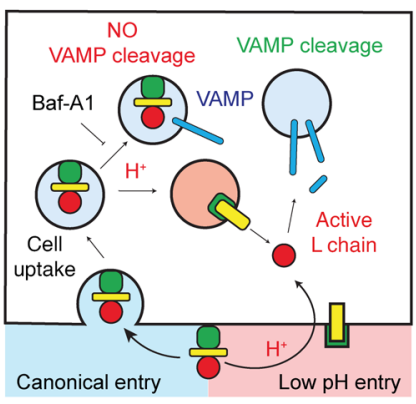

D

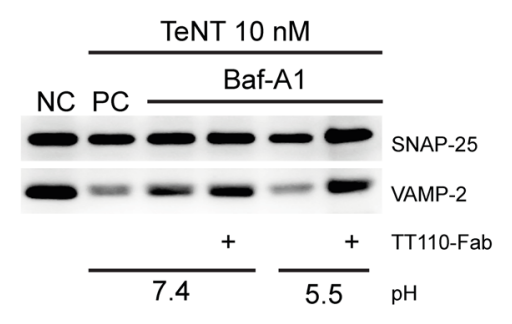

E
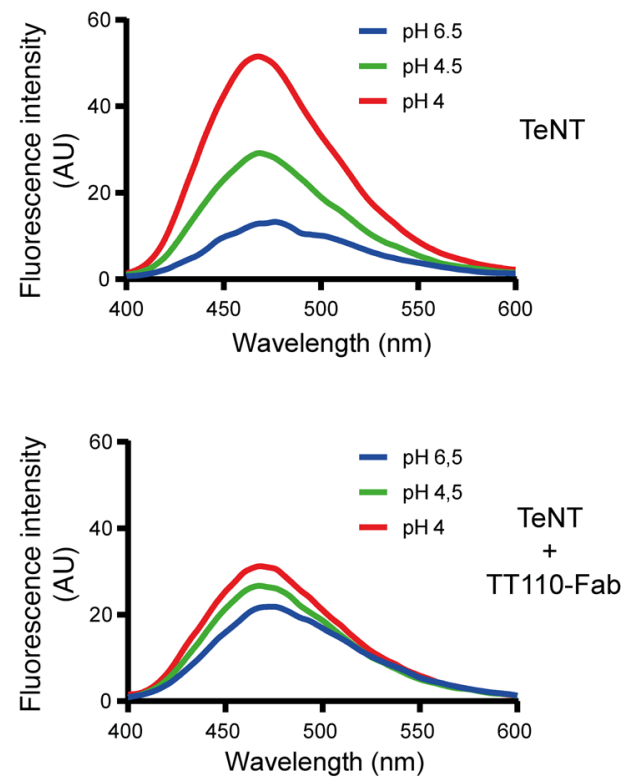

Figure 6. TT110-Fab neutralizes TeNT toxicity by preventing the translocation of the $L$ chain into the nerve terminal cytosol. (A) Western blotting of CGNs treated with 50 pM TeNT alone (PC) or preincubated with the indicated TT104-Fab/TeNT molar ratio. After 12 hours, CGNs were lysed and immunoblotted for VAMP-2 and SNAP-25 as in Figure 2. Blots are representative of 3 independent experiments. (B) Survival curve for mice injected i.p. with either TeNT alone ( $4 \mathrm{ng} / \mathrm{kg}$ ) or premixed with 5 times the molar excess of TT104-Fab. The number of mice in each group is indicated in the panel. (C) Scheme illustrating entry of the TeNT $L$ chain into the neuronal cytosol via either canonical receptor-mediated cell uptake and translocation across the membrane of SVs triggered by the acidification of their lumen induced by proton pump activity of the V-ATPase (light blue), or low-pH translocation across the plasma membrane in the presence of bafilomycin A1 (Baf-A1). (D) Western blot analysis showing the inhibition of TeNT L chain membrane translocation by TT110-Fab. CGNs were incubated at $4^{\circ} \mathrm{C}$ for 15 minutes with either TeNT (10 nM) or TeNT preincubated with TT110-Fab. The culture medium was then replaced with a $37^{\circ} \mathrm{C}$ buffer for 10 minutes at pH 7.4 or pH 5.0. Samples were then incubated for 12 hours with normal medium (PC) or normal medium supplemented with Baf-A1 (100 nM). Membrane translocation was assessed according to VAMP-2 cleavage. SNAP-25 served as a loading control. Results are representative of 3 independent experiments. (E) ANS fluorescence binding experiment showing the $\mathrm{pH}$-induced conformational change of TeNT blocked by TT110-Fab. TeNT $(0.35 \mu \mathrm{M})$ or TeNT preincubated with TT110-Fab was incubated at pH 7.0 in the presence of $50 \mu \mathrm{M}$ ANS and liposomes. The conformational change was triggered by lowering the $\mathrm{pH}$ with sequential addition of specific volumes of $\mathrm{HCl}$ and evaluated following the ANS fluorescence intensity at $470 \mathrm{~nm}$. Results are representative of 2 independent experiments.

er side effects are not uncommon. All of these problems could be overcome by the injection of small amounts of well-characterized $\mathrm{mAbs}$, such as those identified in this study, or even a single mAB, as exemplified by TT104. We believe our findings also pave the way for the use of Fab fragments via the intrathecal route. This procedure was found to produce better results than peripheral injections when the toxin had already undergone retroaxonal transport and had been released into the spinal cord but was strictly limited by the amount of antibody protein that could be safely injected intrathecally $(30,31)$.

The isolation of anti-TeNT humAbs from human B memory cells was recently reported (39-42). TeNT neutralization in mice could be achieved only by preincubation of the toxin with a combination of 3 antibodies and/or using a high molar excess of humAbs with respect to TeNT. As in the present study, the highest neutralizing effect was provided by antibodies specific for the HC binding domain of TeNT. This notion is in agreement with the numerous reports about the activity of mAbs specific for the HC portion of TeNT isolated from mice, or prepared after humanization procedures, or by a scFv antibody phage display library $(61,62)$. Together with our findings with TT104, these data fit with the rather general rule that neutralizing antibodies interfere with the pathogen binding to its receptor. However, it is interesting to note that the strong neutralizing activity of
TT110 indicates that antibodies can also effectively neutralize TeNT by blocking its membrane translocation.

Nine humAbs identified in the present study recognized the HC-N domain, 3 humAbs bound the HC-C domain, and 3 bound the HN domain. These results suggest that the most immunogenic domain of TeNT is HC-N, but this possibility calls for more extensive studies. In addition, the fact that $\mathrm{HC}-\mathrm{N}$-specific humAbs had negligible neutralization activity indicates a structural role for the HC-N domain. This interpretation is in line with the result of Masuyer et al., who showed a direct interaction at low $\mathrm{pH}$ of the $\mathrm{L}$ and HC-C domains, but not with the HC-N (8).

In addition to the biomedical properties of the 2 anti-TeNT humAbs, the structure of the immunocomplex formed by the toxin with the $2 \mathrm{Fab}$ derivatives revealed insights into 2 key steps of the mechanism of nerve terminal intoxication by TeNT. The first insight pertains to the sequential events leading to a productive binding of TeNT to the presynaptic membrane at the NMJ. TeNT binds both a PSG molecule and the proteins nidogen-1/-2, but the sequence of binding events is not known. Nidogens are particularly enriched in the NMJ basal lamina, which enwraps perisynaptic Schwann cells and the nerve terminal, secluding them from the muscle fiber. Nidogens are in a strategic position to capture TeNT molecules entering the NMJ, because they are exposed to 
A
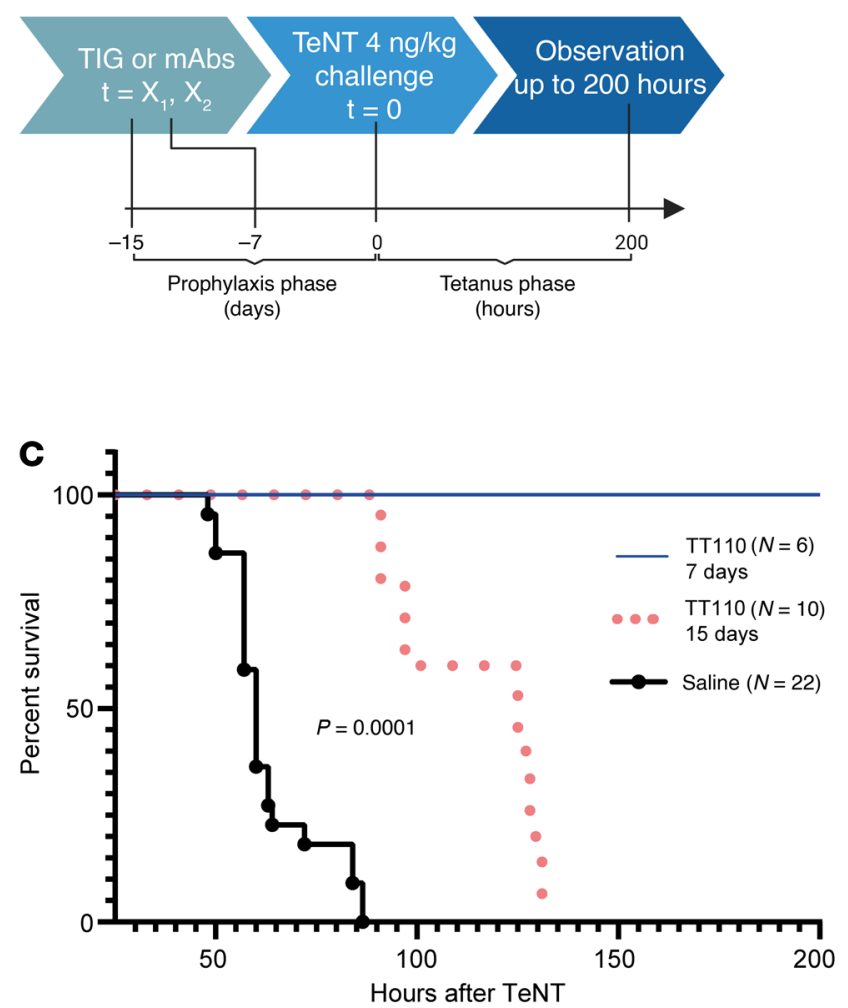

$\mathbf{E}$

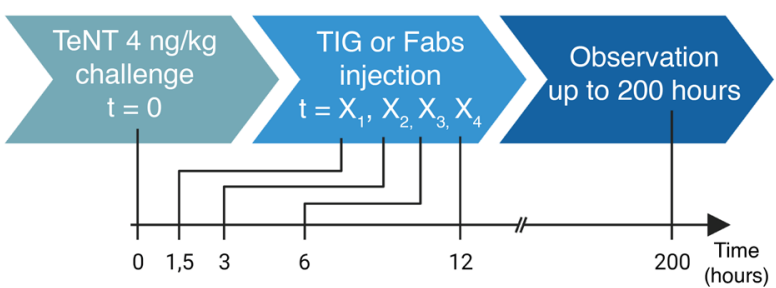

B

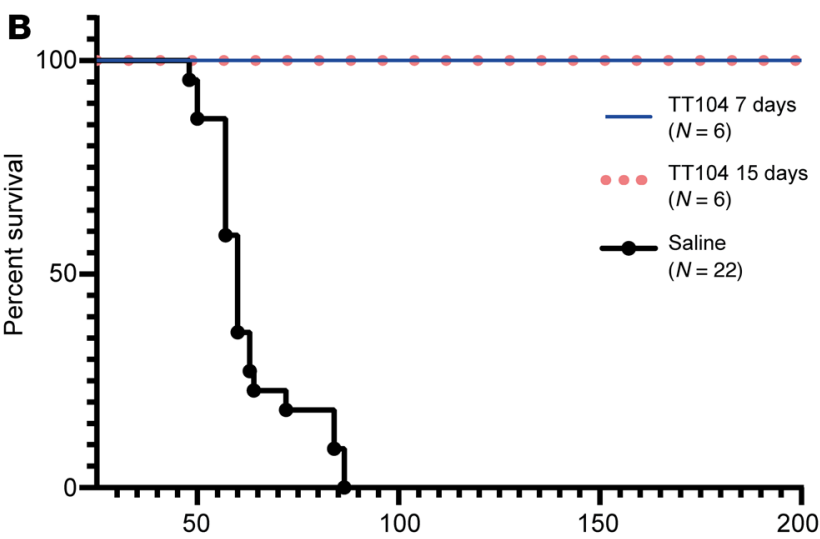

D

Hours after TeNT

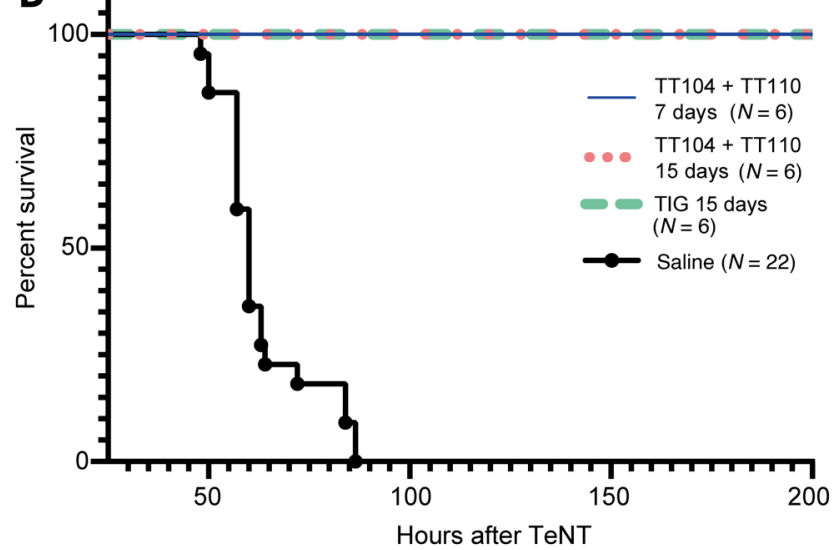

$\mathbf{F}$

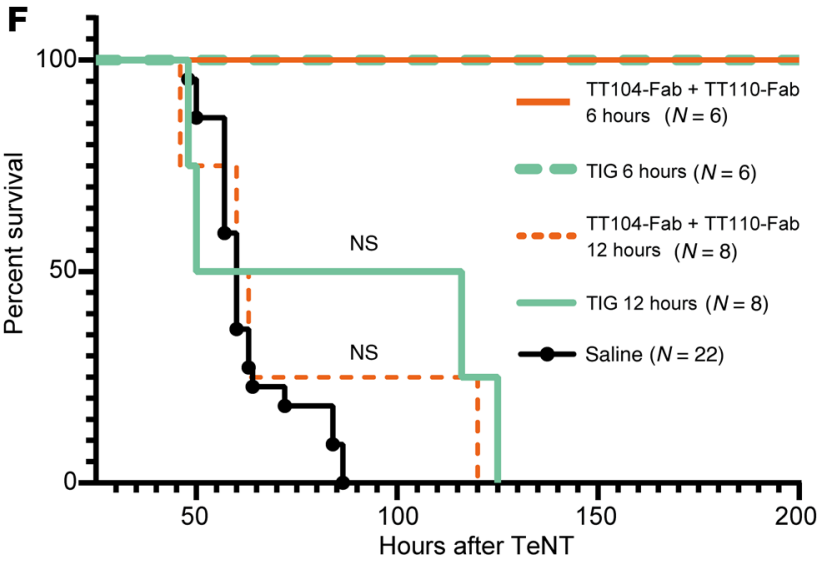

Figure 7. TT104 and TT110 humAbs allow long-lasting prophylactic protection against TeNT, and their Fab derivatives prevent tetanus development after toxin challenge. (A) Time course for testing the prophylactic activity of humAbs. Mice were i.p. preinjected with either TT104 (400 ng/kg) or TT110 ( $400 \mathrm{ng} / \mathrm{kg}$ ) or their combination (200 ng/kg plus $200 \mathrm{ng} / \mathrm{kg}$ ), or with TIG (3.5 IU/kg roughly corresponding to $250 \mathrm{IU} / 70 \mathrm{~kg}$ ) for $15 \mathrm{or} 7 \mathrm{days}$. TeNT (4 ng/kg) was then inoculated i.p., and the animals were observed for 200 hours for tetanus symptoms. (B and C) Prophylactic profiles for TT104 (B) and TT110 (C) injected alone. (D) Survival curves for mice treated with TT104 plus TT110 in combination compared with TIG. (E) Time course for testing TeNT neutralization by Fabs in a post-exposure challenge. TeNT $(4 \mathrm{ng} / \mathrm{kg}$ ) was delivered via i.p. injection. At the indicated time points, the combination of TT104 and TT110 Fab derivatives $(1.2 \mu \mathrm{g} / \mathrm{kg})$ or TIG $(7 \mathrm{IU} / \mathrm{kg})$ was injected i.p., and the animals were observed for 200 hours. (F) Survival plot for mice injected with TeNT and treated with either TT104 plus TT110 Fab derivatives (1.2 $\mathrm{gg} / \mathrm{kg}$, orange traces) or TIG (7 IU/kg, cyan traces) after 6 or 12 hours. Statistical significance was calculated with a Mantel-Cox test. The number of mice in each group is indicated in the panel. B, C, D, and $\mathbf{F}$ display the same lethality curve for the saline group, as this curve was derived from the data for all the mice treated with TeNT alone, plotted together.

the perineural extracellular fluids, yet they are engaged in multiple protein-protein interactions with other basal lamina components, which may limit their availability for TeNT fixation. PSGs are glycolipids located on the outer leaflet of the presynaptic membrane, which are endowed with long and flexible headgroups projecting above the plasma membrane. Our data show that TT104 fully disrupted TeNT interaction with PSG but only partially with nidogens, yet this was sufficient to abrogate TeNT binding and internalization at nerve terminals and to prevent tetanus. This strongly suggests that the interaction with PSG is a prerequisite for TeNT tox- 
Table 1. TeNT neutralization in vivo showing the prophylactic activity of humAbs and Fabs

\begin{tabular}{|c|c|c|c|}
\hline \multicolumn{4}{|l|}{ Prophylactic use } \\
\hline Agent & Dose & Time before TeNT injection & Survival percentage \\
\hline \multirow{2}{*}{ HumAb combination } & \multirow{2}{*}{$400 \mathrm{ng} / \mathrm{kg}$} & $7 d$ & $100 \%$ \\
\hline & & $15 d$ & $100 \%$ \\
\hline
\end{tabular}

HumAbs, TIG, or Fabs were injected at the indicated time points with respect to TeNT inoculation. Survival percentage was calculated for animals surviving up to 200 hours.

icity and that it is the first step of TeNT binding. Nidogens might then drive the TeNT-PSG complex toward the specific endocytic organelle, leading to the formation of signaling endosomes (63).

Our experiments with TT110 provide insights into the mechanism of TeNT entry into nerve terminals. We found that TT110Fab prevented the low-pH-driven conformational change of $\mathrm{HN}$ by binding to an epitope at the center of an important structural-functional module, the BoNT-switch, previously identified in BoNT/A1, which was suggested to be the $\mathrm{pH}$ sensor triggering the initial event of HN membrane insertion (48). The present work indicates that such a structural switch also occurs in TeNT. In addition, the TeNT epitope recognized by TT110 identifies a group of carboxylate residues that are candidates for future studies aimed at determining their role in the TeNT low-pH-triggered switch for membrane insertion.

We believe the results described here qualify the humAbs TT104 and TT110 and their Fab derivatives as therapeutics that are highly superior to the presently used anti-TIGs. These humAbs are ready, after appropriate formulation, to replace the IgGs purified from human or equine blood in clinical practice. Their Fab derivatives open new avenues for the treatment of patients with tetanus, because large doses could be injected intrathecally, thus maximizing the ability of these therapeutics to neutralize TeNT in the very area of its action, i.e., the spinal cord.

\section{Methods}

Additional details can be found in the Supplemental Methods.

HumAbs and Fab fragments. PBMCs $\left(30 \times 10^{6}\right.$ to $50 \times 10^{6}$ cells $)$ were isolated from adult donors who had received a boost dose of tetanus toxoid vaccine at least 1 year before sampling. Memory B cells were isolated from PBMCs using magnetic cell sorting with $0.5 \mu \mathrm{g} / \mathrm{mL}$ anti-CD19-PECy7 antibodies (BD, 341113) and mouse anti-phycoerythrin (anti-PE) microbeads (Miltenyi Biotec, 130-048081), followed by FACS sorting using $3.75 \mu \mathrm{g} / \mathrm{mL}$ Alexa Fluor 647conjugated goat anti-human IgG (Jackson ImmunoResearch, 109606-170), $5 \mu \mathrm{g} / \mathrm{mL}$ Alexa Fluor 647-conjugated goat anti-human IgM (Invitrogen, Thermo Fisher Scientific, A21215), and PE-labeled anti-human IgD (used at 1:40 dilution; BD Biosciences, 555779). Sorted B cells were immortalized with EBV and plated in single-cell cultures in the presence of CpG-DNA $(2.5 \mu \mathrm{g} / \mathrm{mL})$ and irradiated feeder PBMCs, as previously described (35). Two weeks after immortalization, the culture supernatants were tested by ELISA at a 2:5 dilution for binding to TeNT. Briefly, ELISA plates were coated with $1 \mu \mathrm{g} / \mathrm{mL}$ TeNT. The plates were blocked with $1 \%$ BSA and incubated with titrated antibodies, followed by $1 / 500$ alkaline phosphatase-conjugated (AP-conjugated) goat anti-human IgG (Southern Biotech, 2040-04). The plates were then washed, substrate (para-nitrophenyl phosphate [p-NPP], MilliporeSigma) was added, and the plates were read at $405 \mathrm{~nm}$.

Recombinant Fab fragments were produced in HEK-293 cells by introducing a stop codon at the end of $\mathrm{C}_{\mathrm{H}} 1$ and purified by affinity chromatography on an AKTA Xpress Mab System (Cytiva) with UNICORN software, version 5.11 (Build 407), using CaptureSelect $\mathrm{C}_{\mathrm{H}} 1$-XL MiniChrom columns (Thermo Fisher Scientific). Buffer was exchanged for PBS using a HiPrep 26/10 desalting columns (Cytiva). Purified Fabs were concentrated using Amicon Ultra filter units (MilliporeSigma), sterilized through a $0.22 \mathrm{~mm}$ filter, and stored at $-80^{\circ} \mathrm{C}$ after rapid freezing in liquid N2.

Table 2. TeNT neutralization in vivo showing the therapeutic activity of humAbs and Fabs

\begin{tabular}{|c|c|c|c|}
\hline \multicolumn{4}{|l|}{ Therapeutic use } \\
\hline Agent & Dose & Time after TeNT injection & Survival percentage \\
\hline \multirow[b]{2}{*}{ Fab combination } & \multirow[b]{2}{*}{$1.2 \mu \mathrm{g} / \mathrm{kg}$} & $1.5 \mathrm{~h}$ & $100 \%$ \\
\hline & & $3 \mathrm{~h}$ & $100 \%$ \\
\hline \multirow[b]{2}{*}{ TIG } & \multirow{2}{*}{$\begin{array}{l}7 \mathrm{U} / \mathrm{kg} \text { (corresponding to } 1.2 \mu \mathrm{g} / \mathrm{kg} \text { of specific anti-TeNT IgCs } \\
\text { and to } \sim 5 \mathrm{mg} / \mathrm{kg} \text { total proteins) }\end{array}$} & $1.5 \mathrm{~h}$ & $100 \%$ \\
\hline & & $3 \mathrm{~h}$ & $100 \%$ \\
\hline
\end{tabular}

HumAbs, TIG, or Fabs were injected at the indicated time points with respect to TeNT inoculation. Survival percentage was calculated for animals surviving up to 200 hours. 
Cryo-EM data collection and processing. A first screening of the sample and its analysis with cryo-EM (64) suggested strong particle orientation (data not shown). To overcome this problem, data were acquired on a planar orientation and $30^{\circ}$ tilted one. For the planar acquisition, $3 \mathrm{~mL}$ of the sample at $0.5 \mathrm{mg} / \mathrm{mL}$ concentration was applied to the glow-discharged C-flat 2/1-3 Au holey grid and vitrified in a Mark IV Vitrobot (FEI), whereas, for the tilted acquisition, $3 \mathrm{~L}$ of the sample at $0.35 \mathrm{mg} / \mathrm{mL}$ concentration was applied to a glow-discharged Aultrafoil 1.2/1.3 grid. Both grids were imaged with a Titan Krios microscope (Thermo Fisher Scientific) at $300 \mathrm{keV}$ with a K2 direct electron camera at $0.827 \AA$ per pixel. The planar data set was composed of 402 movies (40 frames each and a dose of $1.2 \mathrm{e}^{-} / \AA^{2}$ ) and was combined with the tilted data set containing 2970 movies (50 frames each with $0.68 \mathrm{e}^{-}$/ $\AA^{2}$ ) after beam-induced motion correction with Motioncor2 (65) and contrast transfer function (CTF) estimated using Gctf (66). The selected micrographs were analyzed in RELION-3 (67). An initial model generated with EMAN2 (68) was used for the 3D classification and refinement. An analysis of the 2D classes and the obtained 3D model suggested the presence of some mobility of the domains of the toxin. To overcome this problem, a multibody refinement procedure was performed (69). This refinement gave 12 motion eigenvalues, with the first 3 explaining $85 \%$ of the variance in the data. These eigenvalues allow one to align and subtract the signal of the 2 domains of the protein and to perform a local reconstruction of the 2 main flexible regions $(70,71)$ (see workflow in Supplemental Figure 5). This protocol yielded a $3.9 \AA$ resolution map that matched to the density of the HC domain and the TT104-Fab formed by 98170 particles, and a 8 A low-resolution map of the LC-HN domains bound to TT110-Fab.

HumAb subdomain specificity. TeNT $(0.5 \mu \mathrm{g})$, reduced TeNT $(0.5$ $\mu \mathrm{g}$ in $15 \mathrm{mM}$ DTT), TeNT-HC $(1 \mu \mathrm{g})$, or the individual subdomains (HC-C and HC-N) were separated by SDS-PAGE and transferred onto nitrocellulose membranes. After saturation for 1 hour with $5 \%$ BSA in PBS containing 0.5\% Tween (PBS-T), the membranes were incubated (overnight at $\left.4^{\circ} \mathrm{C}\right)$ with humAbs diluted in PBS-T $(1 \mu \mathrm{g} / \mathrm{mL})$ and used as primary antibodies. After washing, the membranes were incubated for 1 hours with anti-human secondary antibodies conjugated to HRP and then revealed with an Uvitec gel documentation system (Cleaver Scientific) using Luminata Crescendo (MilliporeSigma) as a substrate.

TeNT neutralization assay on primary neuronal cultures. Primary CGNs from 4- to 6-day-old rats were prepared as previously described (44). CGNs at 6-8 days in vitro (DIV) were treated with TeNT (50 pM) alone or preincubated for 1 hour at room temperature (RT) at the indicated molar ratios of TeNT/humAbs or TeNT/Fabs in complete Basal Medium Eagle (BME, Thermo Fisher Scientific). After 12 hours, CGNs were lysed with Laemmli sample buffer containing protease inhibitors (Roche) or fixed for 10 minutes with $4 \%(\mathrm{w} / \mathrm{v})$ paraformaldehyde in PBS. Neutralization was determined by Western blotting to detect the cleavage of intact VAMP-2 (Synaptic System, 104 211), and SNAP-25 (BioLegend, SMI81) was used as a loading control (44).

Fixed CGNs were analyzed by immunofluorescence staining with primary antibodies specific for intact VAMP-2 (Synaptic System, 104211) detected with a fluorescent secondary antibody (44). DAPI was used to stain the nuclei. Coverslips were mounted using Fluorescent Mounting Medium (Dako) and examined with a Leica SP5 confocal microscope (Wetzlar).

Internalization of fluorescent $\mathrm{HC}$ derivatives. BoNT/A-HC- $\mathrm{CpV}(50$ $\mathrm{nM})$ and TeNT-HC-555 (50 nM) were mixed for 1 hour at $37^{\circ} \mathrm{C}$ in com- plete BME with or without TT104-Fab (30 nM), and then added onto 6- to 8-DIV CGNs seeded on glass coverslips. At the indicated time points, the cells were gently rinsed with medium, and incubation was stopped with $4 \%$ paraformaldehyde. The coverslips were extensively washed and then mounted for fluorescence examination with a Leica SP5 confocal microscope.

In mice, $1 \mu \mathrm{g}$ TeNT-HC-555 and BoNT-HC-CpV were mixed in bioassay solution $(0.9 \% \mathrm{NaCl}, 0.2 \%$ gelatin). Then, the solution was split and incubated for 1 hour at $37^{\circ} \mathrm{C}$ with either TT104-Fab (5 molar excess) or the corresponding volume of bioassay solution. A volume of $20 \mu \mathrm{L}$ of either mixture was injected below the skin at the level of the levator auris longus (LAL) of anesthetized CD1 mice. After 2 hours, the LALs were dissected, fixed with $4 \%$ paraformaldehyde, and directly mounted in mounting medium for analysis with a Leica SP5 confocal microscope.

Binding assay for PSG GT1b and nidogens. Ninety-six-well plates (Sarstedt) were coated with either $1 \mu \mathrm{g}$ GT1b (Santa Cruz Biotechnology) dissolved in methanol, or 250 ng nidogen 1/-2 (Bio-Techne) diluted in PBS, or their combination, and allowed to dry overnight at RT. The wells were washed with PBS-T, blocked with $1 \%$ BSA in PBS for 1 hour at RT, and the indicated concentrations of TeNT diluted in PBS, with or without preincubation with TT104-Fab (1 hour at RT), were added for 2 hours at RT. The wells were then extensively washed with PBS-T and incubated with a rabbit TeNT antiserum (Istituto Superiore di Sanità, Rome, Italy) diluted in 1\% BSA in PBS for 1 hour. After washing with PBST, the wells were incubated with the appropriate secondary antibody conjugated with HRP. The wells were extensively washed, and $100 \mu \mathrm{L}$ 2,2'-azino-bis(3-ethylbenzothiazoline-6-sulfonic acid) (Millipore-Sigma) was added. Absorbance was read at $450 \mathrm{~nm}$ with a microplate reader (Tecan).

Low-pH membrane translocation of TeNT in CGNs. CGNs were plated onto 24 -well plates at a density of $4 \times 10^{5}$ cells and incubated in ice-cold complete BME with either $10 \mathrm{nM}$ TeNT or $10 \mathrm{nM}$ TeNT preincubated with $20 \mathrm{nM}$ TT110-Fab. This assay was then performed as described previously (51), and the TeNT L domain activity in the cytosol was evaluated by Western blotting following its VAMP-2specific proteolytic activity described above.

Fluorescence assay of ANS binding to clostridial neurotoxins as a function of $p H$. TeNT alone or TeNT preincubated with 10-fold TT110Fab was diluted to a final concentration of $0.8 \mu \mathrm{M}$ in $100 \mathrm{mM}$ TRIS-citrate buffer and $100 \mathrm{mM} \mathrm{NaCl}, \mathrm{pH}$ 7.0, in the presence of liposomes (final concentration of $0.4 \mathrm{mM}$ ) and $50 \mu \mathrm{M}$ ANS. The assay was then performed as previously described (56).

Mouse bioassay. Swiss-Webster adult CD1 mice (Charles River Laboratories) were housed under controlled 12-hour light/12-hour dark conditions, and food and water were provided ad libitum.

In the preincubation assays, TeNT was diluted at a concentration of $8 \mathrm{pg} / \mu \mathrm{L}$ in bioassay solution, split into aliquots, and supplemented with either an equivolume of bioassay solution (positive control) or an equivolume of bioassay solution supplemented with the indicated amounts of humAbs or Fabs under gentle agitation for 1 hour at RT. Female mice (weighing 24-26 grams) were randomly injected i.p. with $1 \mu \mathrm{L} / \mathrm{g}$ of body weight of either toxin alone or toxin-humAbs/Fabs solutions. The final TeNT dose was $4 \mathrm{ng} / \mathrm{kg}$, roughly corresponding to a 5 -fold lethal dose of our toxin preparation.

For the prophylactic activity, TIG (Igantet, Istituto Grifols Poligono Levante S.A.) or humAbs (TT104 and TT110 alone or their combina- 
tion) were diluted in bioassay solution and i.p. injected at the indicated dose. After 7 or 15 days, TeNT (4 ng/kg) was injected i.p., and mice were monitored for 200 hours, at which point the experiment was terminated. A human endpoint was set at the point at which the mice showed moderate tetanus symptoms (hunched back and paralysis of rear limbs or disappearance of the righting reflex for 30 seconds), after which the animal was euthanized by cervical dislocation and considered positive for tetanus. TIG was used at a dose of $7 \mathrm{IU} / \mathrm{kg}$, roughly corresponding to the canonic prophylactic injection of $500 \mathrm{IU}$ in a human weighing $70 \mathrm{~kg}$.

To test the post-exposure effect of Fabs, TeNT ( $4 \mathrm{ng} / \mathrm{kg}$ ) was i.p. injected. At the indicated time points, either TT104-Fab in combination with TT110-Fab $(1.2 \mu \mathrm{g} / \mathrm{kg})$ or TIG (7 IU/ $/ \mathrm{kg})$ was i.p. injected, and the mice were monitored for 200 hours. The TIG dose was chosen to correspond to the suggested treatment of $500 \mathrm{IU}$ in a human weighing $70 \mathrm{~kg}$. Fab doses were calculated accordingly, estimating the concentration of TeNT-specific IgGs in the serum of a hyperimmunized individual equal to $40 \mu \mathrm{g} / \mathrm{mL}$, as averaged from the values reported previously (57).

Statistics. Statistical analyses were performed with GraphPad Prism (GraphPad Software). Statistical significance in the ELISA assays was calculated using a 2-tailed, unpaired $t$ test. Data are plotted as Kaplan-Meier survival curves, and statistical significance was calculated using the log-rank test. Each curve is representative of at least 6 animals. In each experiment, at least 3 mice were treated with TeNT alone. The survival curves for the mice treated with TeNT alone in Figure 2D and Figure 7, B-D and F, were derived from all the data plotted together. Survival curves were compared using the Mantel-Cox log-rank test. Results were considered significant if the $P$ value was lower than 0.05 .

Data deposition. Atomic coordinates and density maps have been deposited in the PDB as PDB ID 7OHO and EMD-12890 for the HC-TT104-Fab region and PDB ID 7OH1 and EMD-12891 for the L-HN-TT110-Fab region.

Study approval. Our studies were performed in strict accordance with the European Community Council Directive number 2010/63/
UE and approved by the local (University of Padova) and the national ethics committee of the Italian Ministry of Health.

\section{Author contributions}

$\mathrm{CM}, \mathrm{AL}$, and GZ conceptualized the study. MP, AG, OL, FV, MT, SB, DC, CSF, EK, and LO performed experiments. AG and GZ collected and analyzed data. GS, SB, DC, CSF, EK, and LP provided resources. CM, AL, and GZ supervised the study. CM, AL, GZ, GS, and MP wrote the original draft of the manuscript. All authors contributed to the writing, review, and editing of the manuscript.

\section{Acknowledgments}

We are grateful to the European Synchrotron Radiation Facility in Grenoble, France, for providing beam time on the CM01. We thank Gordon A. Leonard for support with data collection and Ornella Rossetto for critical reading of the manuscript. We gratefully acknowledge the financial support of the University of Padova (to MP and CM); the RIPANE project (to CM); the Wellcome Trust (107116/Z/15/Z, to GS); and the UK Dementia Research Institute (UKDRI-1005, to GS).

Address correspondence to: Giuseppe Zanotti, Department of Biomedical Sciences, University of Padova, Via Ugo Bassi 58/B, Padova, 35131, Italy. Phone: 39.049.8276409; Email: giuseppe. zanotti@unipd.it. Or to: Antonio Lanzavecchia, Istituto Nazionale Genetica Molecolare c/o Fondazione IRCCS Cà Granda Ospedale Maggiore Policlinico di Milano, Via Francesco Sforza, 35 - 20122 Milano, Italy. Phone: 39.020.0660300; Email: antonio.lanzavecchia@gmail.com. Or to: Cesare Montecucco, Institute of Neuroscience, National Research Council, Via Ugo Bassi 58/B, Padova, 35131, Italy. Phone:39.049.8276058; Email: cesare.montecucco@ gmail.com. OL's present address is: Paul Scharrer Institute, ETH Villingen, Zurich, Switzerland. FV's present address is: Columbia University, New York, New York, USA. AG's present address is: European Synchrotron Radiation Facility, Grenoble, France. AL's present address is: Humabs BioMed SA, Bellinzona, Switzerland.
1. Cook TM, et al. Tetanus: a review of the literature. Br JAnaesth. 2001;87(3):477-487.

2. Yen LM, Thwaites CL. Tetanus. Lancet. 2019;393(10181):1657-1668.

3. Popoff MR. Tetanus in animals. J Vet Diagn Invest. 2020;32(2):184-191.

4. Rossetto O, Montecucco C. Tables of toxicity of botulinum and tetanus neurotoxins. Toxins (Basel). 2019;11(12):E686.

5. Megighian A, et al. Tetanus and tetanus neurotoxin: From peripheral uptake to central nervous tissue targets. J Neurochem. 2021;158(6):1244-1253.

6. Montecucco C, Schiavo G. Structure and function of tetanus and botulinum neurotoxins. QRev Biophys. 1995;28(4):423-472.

7. Chapeton-Montes D, et al. The population structure of Clostridium tetani deduced from its pan-genome. Sci Rep. 2019;9(1):11220.

8. Masuyer G, et al. The structure of the tetanus toxin reveals $\mathrm{pH}$-mediated domain dynamics. EMBO Rep. 2017;18(8):1306-1317.

9. Bruggemann H, et al. Genomics of clostridium tetani. Res Microbiol. 2015;166(4):326-331.
10. Schiavo G, et al. An intact interchain disulfide bond is required for the neurotoxicity of tetanus toxin. Infect Immun. 1990;58(12):4136-4141.

11. Rummel A, et al. Two carbohydrate binding sites in the H(CC)-domain of tetanus neurotoxin are required for toxicity. $\mathrm{J} \mathrm{Mol} \mathrm{Biol}$. 2003;326(3):835-847.

12. Chen C, et al. Molecular basis for tetanus toxin coreceptor interactions. Biochemistry. 2008;47(27):7179-7186.

13. Chen C, et al. Gangliosides as high affinity receptors for tetanus neurotoxin. J Biol Chem. 2009;284(39):26569-26577.

14. Bercsenyi K, et al. Tetanus toxin entry. Nidogens are therapeutic targets for the prevention of tetanus. Science. 2014;346(6213):1118-1123.

15. Deppe J, et al. The $25 \mathrm{kDa} \mathrm{H}_{\mathrm{CN}}$ domain of clostridial neurotoxins is indispensable for their neurotoxicity. Toxins (Basel). 2020;12(12):E743.

16. Schiavo G, et al. Neurotoxins affecting neuroexocytosis. Physiol Rev. 2000;80(2):717-766.

17. Binz T, Rummel A. Cell entry strategy of clostridial neurotoxins. J Neurochem.
2009;109(6):1584-1595.

18. Deinhardt K, et al. Tetanus toxin is internalized by a sequential clathrin-dependent mechanism initiated within lipid microdomains and independent of epsin1. J Cell Biol. 2006;174(3):459-471.

19. Salinas S, et al. CAR-associated vesicular transport of an adenovirus in motor neuron axons. PLoS Pathog. 2009;5(5):e1000442.

20. Schwab ME, Thoenen H. Electron microscopic evidence for a transsynaptic migration of tetanus toxin in spinal cord motoneurons: an autoradiographic and morphometric study. Brain Res. 1976;105(2):213-227.

21. Matteoli M, et al. Synaptic vesicle endocytosis mediates the entry of tetanus neurotoxin into hippocampal neurons. Proc Natl Acad Sci US A. 1996;93(23):13310-13315.

22. Pirazzini M, et al. The thioredoxin reductasethioredoxin system is involved in the entry of tetanus and botulinum neurotoxins in the cytosol of nerve terminals. FEBS Lett. 2013;587(2):150-155.

23. Schiavo $\mathrm{G}$, et al. Tetanus and botulinum-B neurotoxins block neurotransmitter release by 
proteolytic cleavage of synaptobrevin. Nature. 1992;359(6398):832-835.

24. Jahn R, Scheller RH. SNAREs-engines for membrane fusion. Nat Rev Mol Cell Biol. 2006;7(9):631-643.

25 . Brooks VB, et al. The action of tetanus toxin on the inhibition of motoneurones. J Physiol. 1957;135(3):655-672.

26. Brooks VB, et al. Mode of action of tetanus toxin. Nature. 1955;175(4446):120-121.

27. Amanna IJ, et al. Duration of humoral immunity to common viral and vaccine antigens. $N$ EnglJ Med. 2007;357(19):1903-1915.

28. Thwaites CL, et al. Maternal and neonatal tetanus. Lancet. 2015;385(9965):362-370.

29. Thwaites CL, Loan HT. Eradication of tetanus. Br Med Bull. 2015;116(1):69-77.

30. Miranda-Filho Dde B, et al. Randomised controlled trial of tetanus treatment with antitetanus immunoglobulin by the intrathecal or intramuscular route. BMJ. 2004;328(7440):615.

31. Kabura L, et al. Intrathecal vs. intramuscular administration of human antitetanus immunoglobulin or equine tetanus antitoxin in the treatment of tetanus: a meta-analysis. Trop Med Int Health. 2006;11(7):1075-1081.

32. Nelson AL, et al. Development trends for human monoclonal antibody therapeutics. Nat Rev Drug Discov. 2010;9(10):767-774.

33. Traggiai E, et al. An efficient method to make human monoclonal antibodies from memory B cells: potent neutralization of SARS coronavirus. Nat Med. 2004;10(8):871-875.

34. Lanzavecchia A, et al. Human monoclonal antibodies by immortalization of memory B cells. Curr Opin Biotechnol. 2007;18(6):523-528.

35. Corti D, et al. A neutralizing antibody selected from plasma cells that binds to group 1 and group 2 influenza A hemagglutinins. Science. 2011;333(6044):850-856.

36. Corti D, et al. Protective monotherapy against lethal Ebola virus infection by a potently neutralizing antibody. Science. 2016;351(6279):1339-1342.

37. Pieper K, et al. Public antibodies to malaria antigens generated by two LAIR1 insertion modalities. Nature. 2017;548(7669):597-601.

38. Tan J, et al. A public antibody lineage that potently inhibits malaria infection through dual binding to the circumsporozoite protein. Nat Med. 2018;24(4):401-407.

39. Aliprandini E, et al. An oligoclonal combination of human monoclonal antibodies able to neutralize tetanus toxin in vivo. Toxicon $X$. 2019;2:100006

40. Zhang CM, et al. Structural flexibility of the tetanus neurotoxin revealed by crystallographic and solution scattering analyses. J Struct Biol X. 2021;5:100045

41. Zhang G, et al. Tetanus vaccine-induced human neutralizing antibodies provide full protection against neurotoxin challenge in mice. Int Immunopharmacol. 2021;91:107297.

42. Wang Y, et al. Structural basis of tetanus toxin neutralization by native human monoclonal antibodies. Cell Rep. 2021;35(5):109070.

43. Dong M, et al. Botulinum and tetanus neurotoxins. Annu Rev Biochem. 2019;88:811-837.

44. Azarnia Tehran D, Pirazzini M. Preparation of cerebellum granule neurons from mouse or rat pups and evaluation of clostridial neurotoxin activity and their inhibitors by western blot and immunohistochemistry. Bio Protoc. 2018;8(13):e2918.

45. Umland TC, et al. Structure of the receptor binding fragment $\mathrm{HC}$ of tetanus neurotoxin. Nat Struct Biol. 1997;4(10):788-792.

46. Fotinou $\mathrm{C}$, et al. The crystal structure of tetanus toxin $\mathrm{Hc}$ fragment complexed with a synthetic GT1b analogue suggests cross-linking between ganglioside receptors and the toxin. JBiol Chem. 2001;276(34):32274-32281.

47. Jayaraman $\mathrm{S}$, et al. Common binding site for disialyllactose and tri-peptide in C-fragment of tetanus neurotoxin. Proteins. 2005;61(2):288-295

48. Lam KH, et al. A viral-fusion-peptide-like molecular switch drives membrane insertion of botulinum neurotoxin A1. Nat Commun 2018;9(1):5367.

49.Krissinel E, Henrick K. Inference of macromolecular assemblies from crystalline state. J Mol Biol. 2007;372(3):774-797.

50. Pirazzini M, et al. On the translocation of botulinum and tetanus neurotoxins across the membrane of acidic intracellular compartments. Biochim Biophys Acta. 2016;1858(3):467-474.

51. Pirazzini M, et al. Double anchorage to the membrane and intact inter-chain disulfide bond are required for the low $\mathrm{pH}$ induced entry of tetanus and botulinum neurotoxins into neurons. Cell Microbiol. 2011;13(11):1731-1743.

52. Pirazzini M, et al. Time course and temperature dependence of the membrane translocation of tetanus and botulinum neurotoxins $\mathrm{C}$ and $\mathrm{D}$ in neurons. Biochem Biophys Res Commun. 2013;430(1):38-42.

53. Sun S, et al. Receptor binding enables botulinum neurotoxin $\mathrm{B}$ to sense low $\mathrm{pH}$ for translocation channel assembly. Cell Host Microbe. 2011;10(3):237-247.

54. Gambale F, Montal M. Characterization of the channel properties of tetanus toxin in planar lipid bilayers. Biophys J. 1988;53(5):771-783.

55. Montecucco C, et al. Tetanus toxin is labeled with photoactivatable phospholipids at low pH. Biochemistry. 1986;25(4):919-924.

56. Puhar A, et al. Comparison of the $\mathrm{pH}$-induced conformational change of different clostridial neurotoxins. Biochem Biophys Res Commun. 2004;319(1):66-71.

57. Carrel S, et al. Human tetanus antibodies: Isolation and characterization with special reference to the IgG subclasses. FEBS Lett. 1972;19(4):305-307.

58. Vieira P, Rajewsky K. The half-lives of serum immunoglobulins in adult mice. Eur J Immunol. 1988;18(2):313-316.

59. Mankarious S, et al. The half-lives of IgG subclasses and specific antibodies in patients with primary immunodeficiency who are receiving intravenously administered immunoglobulin. J Lab Clin Med. 1988;112(5):634-640.

60. Hinton PR, et al. Engineered human IgG antibodies with longer serum half-lives in primates. J Biol Chem. 2004;279(8):6213-6216.

61. Wang $\mathrm{H}$, et al. Tetanus neurotoxin neutralizing antibodies screened from a human immune $\mathrm{scFv}$ antibody phage display library. Toxins (Basel). 2016;8(9):266.

62. Ghotloo S, et al. Contribution of Fc fragment of monoclonal antibodies to tetanus toxin neutralization. Neurotox Res. 2020;37(3):578-586.

63. Deinhardt K, et al. Rab5 and Rab7 control endocytic sorting along the axonal retrograde transport pathway. Neuron. 2006;52(2):293-305.

64. Naydenova K, Russo CJ. Measuring the effects of particle orientation to improve the efficiency of electron cryomicroscopy. Nat Commun. 2017;8(1):629.

65. Zheng SQ, et al. MotionCor2: anisotropic correction of beam-induced motion for improved cryo-electron microscopy. Nat Methods. 2017;14(4):331-332.

66. Zhang K. Gctf: real-time CTF determination and correction. J Struct Biol. 2016;193(1):1-12.

67. Zivanov J, et al. New tools for automated high-resolution cryo-EM structure determination in RELION-3. Elife. 2018;7:e42166.

68. Tang G, et al. EMAN2: an extensible image processing suite for electron microscopy. J Struct Biol. 2007;157(1):38-46.

69. Nakane T, et al. Characterisation of molecular motions in cryo-EM single-particle data by multi-body refinement in RELION. Elife. 2018;7:e36861.

70. Ilca SL, et al. Localized reconstruction of subunits from electron cryomicroscopy images of macromolecular complexes. Nat Commun. 2015;6:8843.

71. Zhou Q, et al. Particle segmentation algorithm for flexible single particle reconstruction. Biophys Rep. 2017;3(1):43-55. 